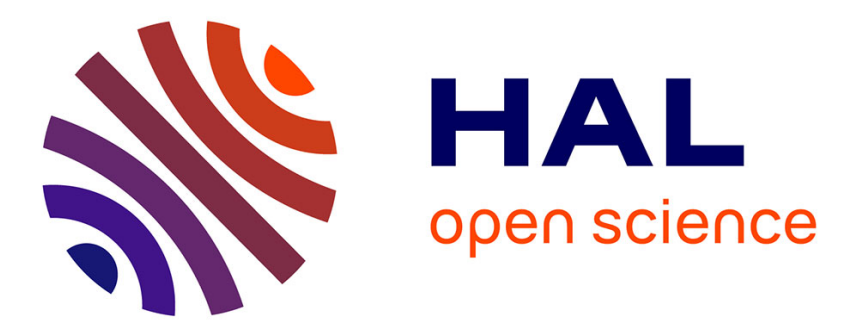

\title{
Frontier estimation with kernel regression on high order moments
}

\author{
Stéphane Girard, Armelle Guillou, Gilles Stupfler
}

\section{To cite this version:}

Stéphane Girard, Armelle Guillou, Gilles Stupfler. Frontier estimation with kernel regression on high order moments. Journal of Multivariate Analysis, 2013, 116, pp.172-189. 10.1016/j.jmva.2012.12.001 . hal-00499369v3

\section{HAL Id: hal-00499369 \\ https://hal.science/hal-00499369v3}

Submitted on 26 Nov 2012

HAL is a multi-disciplinary open access archive for the deposit and dissemination of scientific research documents, whether they are published or not. The documents may come from teaching and research institutions in France or abroad, or from public or private research centers.
L'archive ouverte pluridisciplinaire HAL, est destinée au dépôt et à la diffusion de documents scientifiques de niveau recherche, publiés ou non, émanant des établissements d'enseignement et de recherche français ou étrangers, des laboratoires publics ou privés. 


\title{
Frontier estimation with kernel regression
}

\section{on high order moments}

\author{
Stéphane Girard ${ }^{(1)}$, Armelle Guillou ${ }^{(2)} \&$ Gilles Stupfler ${ }^{(3)}$ \\ (1) Team Mistis, INRIA Rhône-Alpes \& LJK, Inovallée, 655, av. de l'Europe, \\ Montbonnot, 38334 Saint-Ismier cedex, France \\ (2) Université de Strasbourg \& CNRS, IRMA, UMR 7501, 7 rue René Descartes, \\ 67084 Strasbourg cedex, France \\ (3) Université d'Aix-Marseille, CERGAM, 15-19 allée Claude Forbin, \\ 13628 Aix-en-Provence Cedex 1, France
}

\begin{abstract}
We present a new method for estimating the frontier of a multidimensional sample. The estimator is based on a kernel regression on high order moments. It is assumed that the order of the moments goes to infinity while the bandwidth of the kernel goes to zero. The consistency of the estimator is proved under mild conditions on these two parameters. The asymptotic normality is also established when the conditional distribution function decreases at a polynomial rate to zero in the neighborhood of the frontier. The good performance of the estimator is illustrated on some finite sample situations.
\end{abstract}

AMS Subject Classifications: 62G05, 62G20.

Keywords: Frontier estimation, kernel estimation, consistency, asymptotic normality.

\section{Introduction}

Let $\left(X_{1}, Y_{1}\right), \ldots,\left(X_{n}, Y_{n}\right)$ be $n$ independent copies of a random pair $(X, Y)$ such that their common density has a support defined by $S=\{(x, y) \in \Omega \times \mathbb{R} ; 0 \leq y \leq g(x)\}$, where $\Omega$ is a compact subset of $\mathbb{R}^{d}$. The unknown function $g$ is called the frontier. We address the problem of estimating $g$. In Girard and Jacob (2008), an estimator is introduced based upon kernel regression on high power-transformed data. In the particular case where $Y$ given $X=x$ is uniformly distributed it is proved that this estimator is asymptotically Gaussian with the minimax rate of convergence for Lipschitz continuous frontier functions. Compared to the numerous extreme-value based estimators (Gardes (2002), Geffroy (1964), Girard and 
Jacob (2003a, 2003b, 2004), Girard and Menneteau (2005), Menneteau (2008)), projection estimators (Jacob and Suquet (1995)), or piecewise polynomial estimators (Korostelev and Tsybakov (1993), Korostelev et al. (1995), Härdle et al. (1995)) this estimator does not require a partition of the support $S$. When the conditional distribution of $Y$ given $X$ is not uniform, this estimator is still convergent (Girard and Jacob (2008), Theorem 1) but suffers from a strong bias on finite sample situations (Girard and Jacob (2008), Table 1).

Under monotonicity assumptions, the frontier can also be interpreted as the endpoint of $Y$ given $X \leq x$. Specific estimation techniques have been developed in this context, see for instance Deprins et al. (1984), Farrell (1957), Gijbels et al. (1999) or Aragon et al. (2005), Cazals et al. (2002), Daouia and Simar (2005) and Daouia et al. (2012) for the definition and properties of robust estimators. Let us mention that all these techniques apply when there is no random noise in the data. In the presence of noise, a popular and efficient technique consists in using local maximum likelihood estimators, see for instance the pioneering article of Aigner et al. (1976) and its semiparametric, kernel-based generalisations by Fan et al. (1996), Kumbhakar et al. (2007) and Simar and Zelenyuk (2011). Recently, Daouia et al. (2010) have shown that the estimation of the endpoint of $Y$ given $X \leq x$ may be reduced to the estimation of the endpoint of univariate independent and identically distributed random variables. This result tends to indicate that the nature of the problem addressed here is different.

In this paper, an estimator based on a kernel regression on high order moments of the variable of interest $Y$ is introduced. More precisely, the estimator is given by

$$
\frac{1}{\widehat{g}_{n}(x)}=\frac{1}{a p_{n}}\left[\left((a+1) p_{n}+1\right) \frac{\widehat{\mu}_{(a+1) p_{n}}(x)}{\widehat{\mu}_{(a+1) p_{n}+1}(x)}-\left(p_{n}+1\right) \frac{\widehat{\mu}_{p_{n}}(x)}{\widehat{\mu}_{p_{n}+1}(x)}\right]
$$

where $\left(p_{n}\right)$ is a nonrandom sequence such that $p_{n} \rightarrow \infty, a>0$ and

$$
\widehat{\mu}_{p_{n}}(x)=\frac{1}{n} \sum_{i=1}^{n} Y_{i}^{p_{n}} K_{h_{n}}\left(x-X_{i}\right)
$$

is a kernel estimator of the conditional moment $m_{p_{n}}(x)=\mathbb{E}\left(Y^{p_{n}} \mid X=x\right)$. Classically, $K$ is a probability density function on $\mathbb{R}^{d}, K_{h_{n}}(u)=h_{n}^{-d} K\left(u / h_{n}\right)$ and $\left(h_{n}\right)$ is a nonrandom positive sequence such that $h_{n} \rightarrow 0$. Note that $\widehat{\mu}_{p_{n}}(x)$ is the empirical counterpart of the moment $\mu_{p_{n}}(x)=\mathbb{E}\left(Y^{p_{n}} K_{h_{n}}(x-X)\right)$, itself a smoothed version of the conditional moment $m_{p_{n}}(x)$, namely

$$
\mu_{p_{n}}(x)=\int_{\Omega} K_{h_{n}}(x-t) m_{p_{n}}(t) f(t) d t
$$

where $f$ is the probability density function of $X$. From a practical point of view, the use of a small window-width $h_{n}$ allows to select the pairs $\left(X_{i}, Y_{i}\right)$ such that $X_{i}$ is close to $x$ while the use of the high power $p_{n}$ gives more weight to the $Y_{i}$ close to $g(x)$. It appears therefore that our estimator would be sensitive to the presence of noise and especially of outliers. Nevertheless, 
we notice that our estimator does not necessarily envelop all the data points, due to the fact that it is a difference of ratios of high order empirical moments. This property makes our estimator more robust than Geffroy's estimator (1964) or the Free Disposal Hull (Deprins et al., 1984). Moreover, similarly to Girard and Jacob (2008), the kernel regression enables us to avoid the partitioning of $S$. Finally, we highlight that, compared to the estimator suggested in the further work of Girard and Jacob (2008), Section 6, our proposition (1) does not require the knowledge of the conditional extreme-value index. Moreover, it benefits from an explicit formulation which is not the case of estimators defined by optimization problems (Girard et al. (2005)) such as local polynomial estimators (Hall et al. (1998), Hall and Park (2004), Knight (2001)).

The asymptotic properties of the estimator (1) are investigated under two different assumptions. The first one is nonparametric, it is only assumed that

$(N P)$ Given $X=x, Y$ is positive and has a finite right endpoint $g(x)$.

We shall show in Section 3 that, under $(N P)$, the estimator $\widehat{g}_{n}(x)$ converges in probability to $g(x)$ without any parametric assumption neither on the distribution of $X$ nor on the distribution of $Y$ given $X=x$. Remark that, although our estimator $\widehat{g}_{n}(x)$ is based on a kernel regression, classical results do not apply (see for instance Ferraty and Vieu (2005), Theorem 6.11) since the condition $p_{n} \rightarrow \infty$ induces technical difficulties. The second assumption is parametric, the cumulative distribution function of $Y$ given $X=x$ is assumed to be given by

(P) $F(y \mid x)=1-(1-y / g(x))^{\alpha(x)}, \forall y \in[0, g(x)]$.

Here, $\alpha(x)$ is an unknown positive function driving the behavior of the distribution tail of $Y$ given $X=x$ in the neighborhood of its endpoint $g(x)$. If $\alpha(x)<1$ then the density of $Y$ given $X=x$ tends to infinity as $y \rightarrow g(x)$ whereas it tends to 0 in the case $\alpha(x)>1$. The intermediate case $\alpha(x)=1$ corresponds to the uniform distribution already investigated in Girard and Jacob (2008) where the density has a jump at the endpoint. In the general context of extreme-value theory (see for instance Embrechts et al. (1997)), the conditional distribution of $Y$ given $X=x$ is said to belong to the Weibull max-domain of attraction with conditional extreme-value index $-1 / \alpha(x)$. In Section 4 , the estimator is proved to be asymptotically Gaussian under $(P)$. As expected, the asymptotic variance depends on the tail behavior of the conditional distribution of $Y$ given $X=x$ through the quantity $\alpha(x)$. Some simulations are proposed in Section 5 to illustrate the efficiency of our estimator and to compare it with some estimators of the frontier estimation literature, particularly the one 
of Girard and Jacob (2008). Auxiliary results are postponed to Appendix A and proved in Appendix B.

\section{Construction of the estimator}

To motivate the construction of our estimator of $g(x)$, let us first focus on the parametric setting $(P)$. Let $x \in \Omega$ and consider the conditional moment

$$
m_{p_{n}}(x)=p_{n} \int_{0}^{\infty} t^{p_{n}-1} \bar{F}(t \mid x) d t=\alpha(x) g^{p_{n}}(x) B\left(p_{n}+1, \alpha(x)\right)
$$

where $\bar{F}=1-F$ and $B(x, y)=\int_{0}^{1} t^{x-1}(1-t)^{y-1} d t$ is the Beta function. Therefore

$$
\frac{m_{p_{n}}(x)}{m_{p_{n}+1}(x)}=\frac{1}{g(x)}\left(1+\frac{\alpha(x)}{p_{n}+1}\right)
$$

which leads to the equation

$$
\frac{1}{g(x)}=\frac{1}{a p_{n}}\left[\left((a+1) p_{n}+1\right) \frac{m_{(a+1) p_{n}}(x)}{m_{(a+1) p_{n}+1}(x)}-\left(p_{n}+1\right) \frac{m_{p_{n}}(x)}{m_{p_{n}+1}(x)}\right]
$$

for all $a>0$. On the basis of this result, the estimator of $g(x)$ is built in two steps. First, the conditional moment $m_{p_{n}}(x)$ is replaced by its smoothed version $\mu_{p_{n}}(x)$, and we set

$$
\frac{1}{G_{n}(x)}:=\frac{1}{a p_{n}}\left[\left((a+1) p_{n}+1\right) \frac{\mu_{(a+1) p_{n}}(x)}{\mu_{(a+1) p_{n}+1}(x)}-\left(p_{n}+1\right) \frac{\mu_{p_{n}}(x)}{\mu_{p_{n}+1}(x)}\right] .
$$

Second, $\mu_{p_{n}}(x)$ is estimated by the corresponding empirical moment $\widehat{\mu}_{p_{n}}(x)$. Plugging $\widehat{\mu}_{p_{n}}(x)$ in $1 / G_{n}(x)$ leads to the expression (1) of the estimator $1 / \widehat{g}_{n}(x)$ of $1 / g(x)$. In the sequel, it is assumed that

$(K)$ The kernel $K$ is bounded and its support is included in $B$, the unit ball of $\mathbb{R}^{d}$.

Note that $(K)$ implies that $\forall q \geq 1, \int_{B} K^{q}(u) d u<\infty$. The following regularity assumptions are introduced:

$\left(A_{1}\right)$ The conditional survival function $\bar{F}(\cdot \mid x)$ of $Y$ given $X=x$ satisfies

$$
\forall x \in \Omega, \quad \sup _{u \in B}\left|\frac{\int_{0}^{1} y^{p_{n}-1} \bar{F}\left(g\left(x-h_{n} u\right) y \mid x-h_{n} u\right) d y}{\int_{0}^{1} y^{p_{n}-1} \bar{F}(g(x) y \mid x) d y}-1\right| \rightarrow 0 \quad \text { as } n \rightarrow \infty .
$$

$\left(A_{2}\right) f$ is locally Hölder continuous with exponent $\eta_{f}$.

$\left(A_{3}\right) g$ is locally Hölder continuous with exponent $\eta_{g}$.

$\left(A_{4}\right) \alpha$ is locally Hölder continuous with exponent $\eta_{\alpha}$.

Note that assumptions $(P),\left(A_{4}\right)$ and $\log \left(p_{n}\right) h_{n}^{\eta_{\alpha}} \rightarrow 0$ imply $\left(A_{1}\right)$. Finally, for any realvalued function $\gamma$ on $\mathbb{R}^{d}$, the oscillation of $\gamma$ between two points $x$ and $x-h_{n} u, u \in B$, is 
defined by

$$
\Delta_{n}^{\gamma}(x, u)=\gamma\left(x-h_{n} u\right)-\gamma(x)
$$

\section{Consistency}

In this section, the consistency of $\widehat{g}_{n}(x)$ is established in the nonparametric context $(N P)$. To this end, the first step is to prove that (3) still holds, up to an error term, when $m_{p_{n}}(x)$ is replaced by $\mu_{p_{n}}(x)$.

Proposition 1. Let $x \in \Omega$ such that $f(x)>0$, and assume that $(N P),(K)$ and $\left(A_{1}-A_{3}\right)$ hold. If $p_{n} h_{n}^{\eta_{g}} \rightarrow 0$, then

$$
\frac{\mu_{p_{n}}(x)}{\mu_{p_{n}+1}(x)}=\frac{1}{g(x)}(1+\mathrm{o}(1)) .
$$

This result is a straightforward consequence of Lemma 1 . The second step consists in showing that $\mu_{p_{n}}(x)$ can be replaced by its empirical counterpart $\widehat{\mu}_{p_{n}}(x)$. In fact, defining for the sake of simplicity

$$
m_{1, p_{n}}(x)=p_{n} \int_{0}^{1} y^{p_{n}-1} \overline{F_{1}}(y \mid x) d y
$$

where $\overline{F_{1}}(y \mid x):=\bar{F}(g(x) y \mid x)$, a slightly more general result can be established:

Proposition 2. Assume that $(N P),(K)$ and $\left(A_{1}-A_{3}\right)$ are satisfied. Let $x \in \Omega$ such that $f(x)>0$. If $n m_{1, p_{n}}(x) h_{n}^{d} \rightarrow \infty$ and $p_{n} h_{n}^{\eta_{g}} \rightarrow 0$ as $n \rightarrow \infty$, then

$$
\frac{\widehat{\mu}_{p_{n}}(x)}{\mu_{p_{n}}(x)}=1+\mathrm{O} \mathbb{P}(1) .
$$

Proof. Let, for all $1 \leq j \leq n$,

$$
U_{n j}=\frac{Y_{j}^{p_{n}} K_{h_{n}}\left(x-X_{j}\right)}{n \mu_{p_{n}}(x)} .
$$

The desired result is then tantamount to $\sum_{j=1}^{n} U_{n j} \stackrel{\mathbb{P}}{\longrightarrow} 1$ as $n \rightarrow \infty$. Let us highlight that, for all $n$, the $\left(U_{n j}\right)_{1 \leq j \leq n}$ are positive independent random variables, and $\sum_{j=1}^{n} \mathbb{E}\left(U_{n j}\right)=1$. According to Chow and Teicher (1997, Corollary 2 p. 358), it is enough to show that, for all $\varepsilon>0, \sum_{j=1}^{n} \mathbb{E}\left(U_{n j} \mathbb{1}_{\left\{U_{n j} \geq \varepsilon\right\}}\right) \rightarrow 0$ as $n \rightarrow \infty$. Remark that the $U_{n j}$ can be rewritten as

$$
U_{n j}=\frac{V_{n j} K_{h_{n}}\left(x-X_{j}\right)}{n M_{p_{n}}(x)}
$$

where

$$
V_{n j}=\frac{Y_{j}^{p_{n}}}{\max _{u \in B} g^{p_{n}}\left(x-h_{n} u\right)} \text { and } M_{p_{n}}(x)=\frac{\mu_{p_{n}}(x)}{\max _{u \in B} g^{p_{n}}\left(x-h_{n} u\right)} .
$$

The $\left(U_{n j}\right)_{1 \leq j \leq n}$ being identically distributed, it is equivalent to prove that, for all $\varepsilon>0$,

$$
\frac{1}{M_{p_{n}}(x)} \mathbb{E}\left(V_{n 1} K_{h_{n}}(x-X) \mathbb{1}_{\left\{V_{n 1} K_{h_{n}}(x-X) \geq \varepsilon n M_{p_{n}}(x)\right\}}\right) \rightarrow 0 .
$$


Let then $\varepsilon>0$ and notice that

$$
V_{n 1} K_{h_{n}}(x-X) \geq \varepsilon n M_{p_{n}}(x) \Leftrightarrow h_{n}^{d} V_{n 1} K_{h_{n}}(x-X) \geq \varepsilon n M_{p_{n}}(x) h_{n}^{d} .
$$

The left-hand side of the second inequality is positive and bounded by $\max _{\mathbb{R}^{d}} K$. In view of Lemma 1i), condition $n m_{1, p_{n}}(x) h_{n}^{d} \rightarrow \infty$ is equivalent to $n h_{n}^{d} \mu_{p_{n}}(x) / g^{p_{n}}(x) \rightarrow \infty$. Besides, $p_{n} h_{n}^{\eta_{g}} \rightarrow 0$ and (16) in the proof of Lemma 1i) imply that

$$
\max _{u \in B} \frac{g^{p_{n}}\left(x-h_{n} u\right)}{g^{p_{n}}(x)} \rightarrow 1
$$

so that $n M_{p_{n}}(x) h_{n}^{d} \rightarrow \infty$ as $n \rightarrow \infty$. As a consequence, the right-hand side of (5) goes to infinity, so that

$$
\frac{1}{M_{p_{n}}(x)} \mathbb{E}\left(V_{n 1} K_{h_{n}}(x-X) \mathbb{1}_{\left\{h_{n}^{d} V_{n 1} K_{h_{n}}(x-X) \geq \varepsilon n M_{p_{n}}(x) h_{n}^{d}\right\}}\right)=0
$$

eventually, and the result is proved.

As a consequence of the two previous results, we have:

Theorem 1. Suppose that $(N P),(K)$ and $\left(A_{1}-A_{3}\right)$ hold. Let $x \in \Omega$ such that $f(x)>0$. If $n m_{1,(a+1) p_{n}}(x) h_{n}^{d} \rightarrow \infty$ and $p_{n} h_{n}^{\eta_{g}} \rightarrow 0$, then $\widehat{g}_{n}(x) \stackrel{\mathbb{P}}{\longrightarrow} g(x)$ as $n \rightarrow \infty$.

Proof. Note that $m_{1,(a+1) p_{n}}(x) \leq m_{1, p_{n}}(x)$, which implies $n m_{1, p_{n}}(x) h_{n}^{d} \rightarrow \infty$. Thus, Lemma 1ii) entails $n m_{1, p_{n}+1}(x) h_{n}^{d} \rightarrow \infty$ and $n m_{1,(a+1) p_{n}+1}(x) h_{n}^{d} \rightarrow \infty$ as $n \rightarrow \infty$. We can then apply Proposition 2 to rewrite the frontier estimator as:

$$
\frac{1}{\widehat{g}_{n}(x)}=\frac{1}{a p_{n}}\left[\left((a+1) p_{n}+1\right) \frac{\mu_{(a+1) p_{n}}(x)}{\mu_{(a+1) p_{n}+1}(x)}\left(1+\mathrm{O}_{\mathbb{P}}(1)\right)-\left(p_{n}+1\right) \frac{\mu_{p_{n}}(x)}{\mu_{p_{n}+1}(x)}\left(1+\mathrm{O}_{\mathbb{P}}(1)\right)\right] \text {. }
$$

From Proposition 1, we have

$$
\frac{\mu_{p_{n}}(x)}{\mu_{p_{n}+1}(x)} \rightarrow \frac{1}{g(x)} \quad \text { and } \quad \frac{\mu_{(a+1) p_{n}}(x)}{\mu_{(a+1) p_{n}+1}(x)} \rightarrow \frac{1}{g(x)}
$$

as $n \rightarrow \infty$. Replacing in (6), the conclusion follows.

\section{Asymptotic normality}

We now establish the asymptotic distribution of $\widehat{g}_{n}(x)$ under the assumption $(P)$. The parametric model enables us to compute a more precise asymptotic expansion of $\mu_{p_{n}}(x) / \mu_{p_{n}+1}(x)$ than under the nonparametric assumption, see Proposition 1.

Proposition 3. Let $x \in \Omega$ such that $f(x)>0$, and assume that $(P),(K)$ and $\left(A_{2}-A_{4}\right)$ hold. If $p_{n} h_{n}^{\eta_{g}} \rightarrow 0$, then

$$
\frac{\mu_{p_{n}}(x)}{\mu_{p_{n}+1}(x)}=\frac{1}{g(x)}\left[1+\frac{\alpha(x)}{p_{n}+1}\right]+\mathrm{O}\left(h_{n}^{\eta_{g}}+\frac{h_{n}^{\eta_{\alpha}}}{p_{n}}\right) .
$$


Proof. Remark that, retaining notations of Lemma 2, we have

$$
\frac{L_{n}\left(p_{n}+1, x, u\right)}{L_{n}\left(p_{n}, x, u\right)}=1+\frac{\Delta_{n}^{g}(x, u)}{g(x)}-\frac{\Delta_{n}^{\alpha}(x, u)}{p_{n}}+\mathrm{O}\left(\frac{h_{n}^{\eta_{g}}}{p_{n}}+\frac{h_{n}^{\eta_{\alpha}}}{p_{n}^{2}}\right)
$$

uniformly in $u \in B$. Using the expansion of $\mu_{p_{n}}(x)$ provided by Lemma 2ii) with $q=1$ then yields

$$
\begin{aligned}
\frac{\mu_{p_{n}}(x)}{\mu_{p_{n}+1}(x)} & =\frac{1}{g(x)}\left[1+\frac{\alpha(x)}{p_{n}+1}\right] \\
\times & {\left[1+\frac{\int_{B} L_{n}\left(p_{n}, x, u\right)\left[\frac{\Delta_{n}^{\alpha}(x, u)}{p_{n}}-\frac{\Delta_{n}^{g}(x, u)}{g(x)}\right] K(u) d u}{\int_{B} L_{n}\left(p_{n}, x, u\right) K(u) d u}+\mathrm{O}\left(\frac{h_{n}^{\eta_{g}}}{p_{n}}+\frac{h_{n}^{\eta_{\alpha}}}{p_{n}^{2}}\right)\right] . }
\end{aligned}
$$

To conclude, from Lemma $2 \mathrm{i}), L_{n}\left(p_{n}, x, u\right) \rightarrow 1$ as $n \rightarrow \infty$ uniformly in $u \in B$ so that

$$
\frac{\int_{B} L_{n}\left(p_{n}, x, u\right)\left[\frac{\Delta_{n}^{\alpha}(x, u)}{p_{n}}-\frac{\Delta_{n}^{g}(x, u)}{g(x)}\right] K(u) d u}{\int_{B} L_{n}\left(p_{n}, x, u\right) K(u) d u}=\mathrm{O}\left(h_{n}^{\eta_{g}}+\frac{h_{n}^{\eta_{\alpha}}}{p_{n}}\right)
$$

which entails

$$
\frac{\mu_{p_{n}}(x)}{\mu_{p_{n}+1}(x)}=\frac{1}{g(x)}\left[1+\frac{\alpha(x)}{p_{n}+1}\right]+\mathrm{O}\left(h_{n}^{\eta_{g}}+\frac{h_{n}^{\eta_{\alpha}}}{p_{n}}\right)
$$

and completes the proof of Proposition 3.

As a straightforward consequence, we obtain a control of the bias introduced by replacing $m_{p_{n}}(x)$ by $\mu_{p_{n}}(x)$. If $p_{n} h_{n}^{\eta_{g}} \rightarrow 0$, then

$$
\frac{1}{G_{n}(x)}=\frac{1}{g(x)}+\mathrm{O}\left(h_{n}^{\eta_{g}}+\frac{h_{n}^{\eta_{\alpha}}}{p_{n}}\right) .
$$

Let us now turn to the random term:

Theorem 2. Suppose $(P),(K)$ and $\left(A_{2}-A_{4}\right)$ hold. Let $x \in \Omega$ such that $f(x)>0$. If $n p_{n}^{-\alpha(x)} h_{n}^{d} \rightarrow \infty$ and $p_{n} h_{n}^{\eta_{g}} \rightarrow 0$ then

$$
v_{n}(x)\left(\frac{\widehat{g}_{n}(x)}{G_{n}(x)}-1\right) \stackrel{d}{\longrightarrow} \mathcal{N}\left(0, \frac{\|K\|_{2}^{2} V(\alpha(x), a)}{f(x)}\right), \quad \text { as } n \rightarrow \infty
$$

where $v_{n}(x)=\sqrt{n} p_{n}^{-\alpha(x) / 2+1} h_{n}^{d / 2},\|K\|_{2}^{2}=\int_{B} K^{2}(x) d x$ and

$$
V(\alpha(x), a)=\frac{\alpha(x)+1}{a^{2} \Gamma(\alpha(x))}\left[2^{-\alpha(x)-2}-2 \frac{(a+1)^{\alpha(x)+1}}{(a+2)^{\alpha(x)+2}}+2^{-\alpha(x)-2}(a+1)^{\alpha(x)}\right] .
$$

Proof. Our goal is to prove that the sequence of random variables

$$
\xi_{n}(x)=\frac{g(x)}{\|K\|_{2}} \sqrt{\frac{f(x)}{V(\alpha(x), a)}} \cdot v_{n}(x)\left(\frac{1}{\widehat{g}_{n}(x)}-\frac{1}{G_{n}(x)}\right)
$$

converges in distribution to a standard Gaussian random variable. The first step consists to use Lemma 3 in order to linearize $\xi_{n}(x)$ :

$$
\begin{aligned}
\xi_{n}(x) & =\left[\zeta_{n}^{(1)}(x)+\left(\frac{\mu_{p_{n}+1}(x)}{\widehat{\mu}_{p_{n}+1}(x)}-1\right) \zeta_{n}^{(2)}(x)+\left(1+\frac{a p_{n}}{p_{n}+1}\right)\left(\frac{\mu_{(a+1) p_{n}+1}(x)}{\widehat{\mu}_{(a+1) p_{n}+1}(x)}-1\right) \zeta_{n}^{(3)}(x)\right] \\
& \times u_{n, a}(x)(1+\mathrm{o}(1)) .
\end{aligned}
$$


Now, Proposition 2 yields

$$
\xi_{n}(x)=u_{n, a}(x)\left[\zeta_{n}^{(1)}(x)+\mathrm{o}_{\mathbb{P}}\left(\zeta_{n}^{(2)}(x)\right)+\mathrm{o} \mathbb{P}\left(\zeta_{n}^{(3)}(x)\right)\right](1+\mathrm{o}(1))
$$

and to conclude the proof, it is sufficient to establish that

$$
\begin{aligned}
& u_{n, a}(x) \zeta_{n}^{(1)}(x) \stackrel{d}{\longrightarrow} \mathcal{N}(0,1), \\
& u_{n, a}(x) \zeta_{n}^{(2)}(x) \stackrel{d}{\longrightarrow} \mathcal{N}\left(0, C_{2}\right), \\
& u_{n, a}(x) \zeta_{n}^{(3)}(x) \stackrel{d}{\longrightarrow} \mathcal{N}\left(0, C_{3}\right),
\end{aligned}
$$

where $C_{2}$ and $C_{3}$ are positive constants. Note that in fact, (8b) and (8c) are stronger than what is necessary, but their proofs are similar to $(8 \mathrm{a})$. In all the sequel, we set: $Z_{k}^{(n, c, j)}(x)=Y_{k}^{c p_{n}+j} K_{h_{n}}\left(x-X_{k}\right)$, so that $\mu_{c p_{n}+j}(x)=\mathbb{E}\left(Z^{(n, c, j)}(x)\right)$. To prove (8a), remark that $\zeta_{n}^{(1)}(x)$ can be expanded as the sum of independent and centered random variables: $\zeta_{n}^{(1)}(x)=\sum_{k=1}^{n} S_{n, k}^{(1)}(x)$ with

$$
\begin{aligned}
S_{n, k}^{(1)}(x) & =\frac{1}{n}\left[Z_{k}^{(n, 1,0)}(x), Z_{k}^{(n, 1,1)}(x), Z_{k}^{(n, a+1,0)}(x), Z_{k}^{(n, a+1,1)}(x)\right] A_{n}^{(1)}(x), \\
A_{n}^{(1)}(x) & =\left[a_{n, 0}^{(1)}(x), a_{n, 1}^{(1)}(x), a_{n, 2}^{(1)}(x), a_{n, 3}^{(1)}(x)\right]^{t}, \\
a_{n, 0}^{(1)}(x) & =-1 \\
a_{n, 1}^{(1)}(x) & =\frac{\mu_{p_{n}}(x)}{\mu_{p_{n}+1}(x)} \\
a_{n, 2}^{(1)}(x) & =\left(1+\frac{a p_{n}}{p_{n}+1}\right) \frac{\mu_{p_{n}+1}(x)}{\mu_{(a+1) p_{n}+1}(x)}, \\
a_{n, 3}^{(1)}(x) & =-\left(1+\frac{a p_{n}}{p_{n}+1}\right) \frac{\mu_{p_{n}+1}(x) \mu_{(a+1) p_{n}}(x)}{\mu_{(a+1) p_{n}+1}^{2}(x)},
\end{aligned}
$$

where $A^{t}$ stands for the transposed matrix of $A$. In order to use Lyapounov's central limit theorem (see e.g. Billingsley, 1979, p. 312), it remains to prove that

$$
\frac{1}{\left[\operatorname{Var}\left(\zeta_{n}^{(1)}(x)\right)\right]^{3 / 2}} \sum_{k=1}^{n} \mathbb{E}\left|S_{n, k}^{(1)}(x)\right|^{3} \rightarrow 0
$$

as $n \rightarrow \infty$, which requires to control $\operatorname{Var}\left(\zeta_{n}^{(1)}(x)\right)$ and $\mathbb{E}\left|S_{n, k}^{(1)}(x)\right|^{3}$. The variance can be rewritten as

$$
\begin{aligned}
n \operatorname{Var}\left(\zeta_{n}^{(1)}(x)\right) & =w\left(p_{n}, p_{n}\right)(x)-2\left(1+\frac{a p_{n}}{p_{n}+1}\right) \frac{\mu_{p_{n}+1}(x)}{\mu_{(a+1) p_{n}+1}(x)} w\left(p_{n},(a+1) p_{n}\right)(x) \\
& +\left(1+\frac{a p_{n}}{p_{n}+1}\right)^{2} \frac{\mu_{p_{n}+1}^{2}(x)}{\mu_{(a+1) p_{n}+1}^{2}(x)} w\left((a+1) p_{n},(a+1) p_{n}\right)(x)
\end{aligned}
$$

where

$$
w\left(s p_{n}+t, u p_{n}+v\right)(x)=\left[-1, \frac{\mu_{s p_{n}+t}(x)}{\mu_{s p_{n}+t+1}(x)}\right] \mathcal{M}_{n}(s, t, u, v)(x)\left[-1, \frac{\mu_{u p_{n}+v}(x)}{\mu_{u p_{n}+v+1}(x)}\right]^{t}
$$

and $\mathcal{M}_{n}(s, t, u, v)(x)$ is the $2 \times 2$ covariance matrix defined by

$$
\mathcal{M}_{n}(s, t, u, v)(x)=\left[\begin{array}{cc}
\mathbb{E}\left(Z^{(n, s, t)}(x) Z^{(n, u, v)}(x)\right) & \mathbb{E}\left(Z^{(n, s, t)}(x) Z^{(n, u, v+1)}(x)\right) \\
\mathbb{E}\left(Z^{(n, s, t+1)}(x) Z^{(n, u, v)}(x)\right) & \mathbb{E}\left(Z^{(n, s, t+1)}(x) Z^{(n, u, v+1)}(x)\right)
\end{array}\right] .
$$


Since Lemma 2iii) provides an asymptotic expansion of the matrix $\mathcal{M}_{n}(s, t, u, v)(x)$, it is therefore sufficient to compute an asymptotic expansion of $\mu_{s p_{n}+t}(x) / \mu_{s p_{n}+t+1}(x)$. Using Proposition 3 and tedious computations lead to

$$
\operatorname{Var}\left(\zeta_{n}^{(1)}(x)\right)=a^{2}\|K\|_{2}^{2} f(x) \Gamma^{2}(\alpha(x)+1) V(\alpha(x), a) \frac{1}{n} \frac{1}{h_{n}^{d}} g^{2 p_{n}}(x) p_{n}^{-\alpha(x)-2}(1+\mathrm{o}(1)) .
$$

Now, focusing on the third moment, Hölder's inequality yields

$$
\begin{aligned}
n^{3} \mathbb{E}\left|S_{n, 1}^{(1)}(x)\right|^{3} & \leq 4 \mathbb{E}\left|a_{n, 0}^{(1)}(x) Z_{1}^{(n, 1,0)}(x)+a_{n, 1}^{(1)}(x) Z_{1}^{(n, 1,1)}(x)\right|^{3} \\
& +4 \mathbb{E}\left|a_{n, 2}^{(1)}(x) Z_{1}^{(n, a+1,0)}(x)+a_{n, 3}^{(1)}(x) Z_{1}^{(n, a+1,1)}(x)\right|^{3} .
\end{aligned}
$$

The next step consists in applying Lemma 4 to each term of the right-hand side of this inequality. To this end, let us consider the functions

$$
\begin{aligned}
& H_{n, 0}^{(1)}(u)=-1 \\
& H_{n, 1}^{(1)}(u)=\alpha(x) u \\
& H_{n, 2}^{(1)}(u)=\left(1+\frac{a p_{n}}{p_{n}+1}\right) g^{a p_{n}}(x) \frac{\mu_{p_{n}+1}(x)}{\mu_{(a+1) p_{n}+1}(x)}, \\
& H_{n, 3}^{(1)}(u)=-\left(1+\frac{a p_{n}}{p_{n}+1}\right) g^{a p_{n}}(x) \frac{\mu_{p_{n}+1}(x)}{\mu_{(a+1) p_{n}+1}(x)} \cdot \frac{\alpha(x) u}{a+1},
\end{aligned}
$$

and note that there exist two sequences of measurable functions $\left(\chi_{n, 1}\right)$ and $\left(\chi_{n, 2}\right)$ uniformly convergent to 0 on $[0,1]$ such that

$$
\begin{aligned}
\max _{u \in B}\left|a_{n, 0}^{(1)}(x)+a_{n, 1}^{(1)}(x) g\left(x-h_{n} u\right) y\right| & \leq\left|H_{n, 0}^{(1)}(y)\right|(1-y)+\frac{\left|H_{n, 1}^{(1)}(y)\right|+\chi_{n, 1}(y)}{p_{n}}, \\
\max _{u \in B}\left|a_{n, 2}^{(1)}(x)+a_{n, 3}^{(1)}(x) g\left(x-h_{n} u\right) y\right| & \leq \frac{1}{g^{a p_{n}}(x)}\left[\left|H_{n, 2}^{(1)}(y)\right|(1-y)+\frac{\left|H_{n, 3}^{(1)}(y)\right|+\chi_{n, 2}(y)}{p_{n}}\right] .
\end{aligned}
$$

Since $g^{a p_{n}}(x) \mu_{p_{n}+1}(x) / \mu_{(a+1) p_{n}+1}(x) \rightarrow(a+1)^{\alpha(x)}$ as $n \rightarrow \infty$, the functions $H_{n, j}^{(1)}, j \in$ $\{0,1,2,3\}$ are bounded on $[0,1]$, uniformly in $n$, and thus Lemma 4 entails that

$$
\mathbb{E}\left|S_{n, 1}^{(1)}(x)\right|^{3}=\mathrm{O}\left(n^{-3} g^{3 p_{n}}(x) p_{n}^{-\alpha(x)-3} h_{n}^{-2 d}\right) .
$$

Combining (11) and (12), convergence (10) follows from the condition $n p_{n}^{-\alpha(x)} h_{n}^{d} \rightarrow \infty$ and therefore (8a) holds.

Proofs of (8b) and (8c) are similar since $\zeta_{n}^{(2)}$ and $\zeta_{n}^{(3)}$ can be rewritten as

$$
\begin{aligned}
\zeta_{n}^{(2)}(x) & =\frac{1}{n} \sum_{k=1}^{n}\left[Z_{k}^{(n, 1,0)}(x), Z_{k}^{(n, 1,1)}(x)\right]\left[a_{n, 0}^{(2)}(x), a_{n, 1}^{(2)}(x)\right]^{t} \\
\zeta_{n}^{(3)}(x) & =\frac{1}{n} \sum_{k=1}^{n}\left[Z_{k}^{(n, a+1,0)}(x), Z_{k}^{(n, a+1,1)}(x)\right]\left[a_{n, 0}^{(3)}(x), a_{n, 1}^{(3)}(x)\right]^{t}
\end{aligned}
$$


with clear definitions of the sequences $a_{n, i}^{(j)}(x), i=0,1, j=2,3$. Applying Lemma 4 with

$$
\begin{aligned}
& H_{n, 0}^{(2)}(u)=-1, \\
& H_{n, 1}^{(2)}(u)=\alpha(x) u \\
& H_{n, 0}^{(3)}(u)=g^{a p_{n}}(x) \frac{\mu_{p_{n}+1}(x)}{\mu_{(a+1) p_{n}+1}(x)}, \\
& H_{n, 1}^{(3)}(u)=-g^{a p_{n}}(x) \frac{\mu_{p_{n}+1}(x)}{\mu_{(a+1) p_{n}+1}(x)} \cdot \frac{\alpha(x) u}{a+1}
\end{aligned}
$$

yields $\mathbb{E}\left|S_{n, 1}^{(j)}(x)\right|^{3}=\mathrm{O}\left(n^{-3} g^{3 p_{n}}(x) p_{n}^{-\alpha(x)-3} h_{n}^{-2 d}\right), j=2,3$. Lyapounov's central limit theorem then gives the convergence. Theorem 2 is therefore established.

From the expansion

$$
\widehat{g}_{n}(x)-g(x)=G_{n}(x)\left[\frac{\widehat{g}_{n}(x)}{G_{n}(x)}-1\right]+\left[G_{n}(x)-g(x)\right],
$$

the asymptotic normality of $\widehat{g}_{n}(x)$ centered on the true function $g(x)$ is readily obtained:

Theorem 3. Suppose $(P),(K)$ and $\left(A_{2}-A_{4}\right)$ hold. Let $x \in \Omega$ such that $f(x)>0$. If $n p_{n}^{-\alpha(x)} h_{n}^{d} \rightarrow \infty, n p_{n}^{-\alpha(x)+2} h_{n}^{d+2 \eta_{g}} \rightarrow 0$ and $n p_{n}^{-\alpha(x)} h_{n}^{d+2 \eta_{\alpha}} \rightarrow 0$, then

$$
v_{n}(x)\left(\frac{\widehat{g}_{n}(x)}{g(x)}-1\right) \stackrel{d}{\longrightarrow} \mathcal{N}\left(0, \frac{\|K\|_{2}^{2} V(\alpha(x), a)}{f(x)}\right), \quad \text { as } n \rightarrow \infty \text {. }
$$

Let us note that $n p_{n}^{-\alpha(x)} h_{n}^{d} \rightarrow \infty$ and $n p_{n}^{-\alpha(x)+2} h_{n}^{d+2 \eta_{g}} \rightarrow 0$ imply that $p_{n} h_{n}^{\eta_{g}} \rightarrow 0$. Besides, if we assume that $\alpha$ has greater regularity than $g$, namely $\eta_{\alpha} \geq \eta_{g}$, then the hypotheses necessary to apply Theorem 3 can be reduced to $n p_{n}^{-\alpha(x)} h_{n}^{d} \rightarrow \infty$ and $n p_{n}^{-\alpha(x)+2} h_{n}^{d+2 \eta_{g}} \rightarrow 0$. Let $x \in \Omega$ such that $f(x)>0$ and note that the sequences

$$
h_{n}(x)=\varepsilon_{n}^{\alpha(x)-1} n^{-1 /\left(d+\eta_{g} \alpha(x)\right)} \quad \text { and } \quad p_{n}(x)=\varepsilon_{n}^{d+\eta_{g}} n^{\eta_{g} /\left(d+\eta_{g} \alpha(x)\right)}
$$

can be chosen to check the assumptions of Theorem 3 , where $\left(\varepsilon_{n}\right)$ is an arbitrary sequence of positive real numbers tending to 0 such that $n^{-\delta} \varepsilon_{n} \rightarrow 0$ for all $\delta>0$. With such choices, the rate of convergence $v_{n}(x)$ of the estimator is then $n^{\eta_{g} /\left(d+\eta_{g} \alpha(x)\right)}$ up to a $\varepsilon_{n}$ term. In the uniform case (that is, when $\alpha$ is constant equal to 1 ), the rate of convergence of the estimator is then $n^{\eta_{g} /\left(d+\eta_{g}\right)}$, up to the factor $\varepsilon_{n}$, which is also the rate of convergence for the Parzen estimator studied in Girard and Jacob (2008), Theorem 2. Let us note that this rate of convergence has been shown to be minimax by Härdle et al. (1995) for a particular class of densities with a $L^{1}$ risk. Clearly, the rate of convergence is a decreasing function of the dimension $d$ of the covariate $X$. This is often referred to as the "curse of dimensionality" effect for nonparametric estimators. This problem may be overcome using semi-parametric dimension reduction techniques, see for instance Härdle and Stoker (1989).

The asymptotic variance of the estimator also involves the multiplicative factor $V(\alpha(x), a)$. The choice of an "optimal" value for $a$ by minimization of $V(\alpha(x), a)$ is a difficult task since 
it depends on the unknown value of $\alpha(x)$. One can observe on Figure 1 that, for $\alpha(x) \leq 2$, $V(\alpha(x), \cdot)$ is a decreasing function and thus large values of $a$ should be preferred.

However, both statements above require a precise knowledge of the function $x \mapsto \alpha(x)$, which is often unrealistic. In view of these remarks, it may be of interest to estimate $\alpha(x)$. From (3), the following estimator is considered:

$$
\widehat{\alpha}_{n}(x)=\left(p_{n}+1\right)\left[\widehat{g}_{n}(x) \frac{\widehat{\mu}_{p_{n}}(x)}{\widehat{\mu}_{p_{n}+1}(x)}-1\right],
$$

and its weak consistency is established under the same assumptions as in Theorem 3.

Proposition 4. Under the assumptions of Theorem 3, $\widehat{\alpha}_{n}(x)=\alpha(x)+\mathrm{O}_{\mathbb{P}}\left(p_{n} / v_{n}(x)\right)$.

Proof. Define

$$
\alpha_{n}(x)=\left(p_{n}+1\right)\left[G_{n}(x) \frac{\mu_{p_{n}}(x)}{\mu_{p_{n}+1}(x)}-1\right]
$$

and let us focus first on the random term

$\frac{v_{n}(x)}{p_{n}}\left(\widehat{\alpha}_{n}(x)-\alpha_{n}(x)\right)=v_{n}(x)\left[\left[\widehat{g}_{n}(x)-G_{n}(x)\right] \frac{\widehat{\mu}_{p_{n}}(x)}{\widehat{\mu}_{p_{n}+1}(x)}-G_{n}(x) \frac{\mu_{p_{n}+1}(x)}{\widehat{\mu}_{p_{n}+1}(x)} \cdot \frac{\zeta_{n}^{(2)}(x)}{\mu_{p_{n}+1}(x)}\right](1+\mathrm{o}(1))$

with notations of Lemma 3. Recall that, from Proposition $1, \mu_{p_{n}}(x) / \mu_{p_{n}+1}(x) \rightarrow 1 / g(x)$, from Proposition $2, \mu_{p_{n}}(x) / \widehat{\mu}_{p_{n}}(x) \stackrel{\mathbb{P}}{\longrightarrow} 1$ and from $(7), G_{n}(x) \rightarrow g(x)$ as $n \rightarrow \infty$ so that $\frac{v_{n}(x)}{p_{n}}\left(\widehat{\alpha}_{n}(x)-\alpha_{n}(x)\right)=v_{n}(x)\left[\left(\widehat{g}_{n}(x)-G_{n}(x)\right)\left[\frac{1}{g(x)}+\mathrm{O}_{\mathbb{P}}(1)\right]-g(x) \frac{\zeta_{n}^{(2)}(x)}{\mu_{p_{n}+1}(x)}(1+\mathrm{O} \mathbb{P}(1))\right]$.

Besides, applying Theorem 2 yields $v_{n}(x)\left(\widehat{g}_{n}(x)-G_{n}(x)\right)=\mathrm{O}_{\mathbb{P}}(1)$. Now,

$$
v_{n}(x) \frac{\zeta_{n}^{(2)}(x)}{\mu_{p_{n}+1}(x)}=\frac{v_{n}(x)}{\mu_{p_{n}+1}(x) u_{n, a}(x)} u_{n, a}(x) \zeta_{n}^{(2)}(x)=\mathrm{O}_{\mathbb{P}}(1),
$$

from $(21)$ and since $u_{n, a}(x) \zeta_{n}^{(2)}(x)$ is asymptotically Gaussian (see (8b)). As a preliminary conclusion, we have

$$
\frac{v_{n}(x)}{p_{n}}\left(\widehat{\alpha}_{n}(x)-\alpha_{n}(x)\right)=\mathrm{O}_{\mathbb{P}}(1) .
$$

Turning to the bias term, (7) and Proposition 3 yield

$$
\alpha_{n}(x)=\alpha(x)+\left(p_{n}+1\right) \mathrm{O}\left(h_{n}^{\eta_{g}}+\frac{h_{n}^{\eta_{\alpha}}}{p_{n}}\right)=\alpha(x)+\mathrm{o}\left(p_{n} / v_{n}(x)\right),
$$

which completes the proof.

Meanwhile, the density function $f(x)$ can be estimated with the classical kernel estimator:

$$
\widehat{f}_{n}(x)=\frac{1}{n} \sum_{i=1}^{n} K_{h_{n}}\left(x-X_{i}\right)
$$

Since Parzen (1962), it is well-known that $\widehat{f}_{n}(x) \stackrel{\mathbb{P}}{\longrightarrow} f(x)$ when $n h_{n}^{d} \rightarrow \infty$. By plugging $\widehat{\alpha}_{n}(x)$ and $\widehat{f}_{n}(x)$ in the asymptotic variance of Theorem 3 , classical arguments thus yield: 
Corollary 1. Under the assumptions of Theorem 3,

$$
v_{n}(x) \sqrt{\frac{\widehat{f}_{n}(x)}{V\left(\widehat{\alpha}_{n}(x), a\right)}}\left(\frac{\widehat{g}_{n}(x)}{g(x)}-1\right) \stackrel{d}{\longrightarrow} \mathcal{N}\left(0,\|K\|_{2}^{2}\right), \quad \text { as } n \rightarrow \infty .
$$

Pointwise confidence intervals for the frontier may then be built using this result.

\section{Numerical experiments}

The behavior of the proposed frontier estimator is investigated on different situations. In particular, we examine the case $d=1$ where $X$ is uniformly distributed on $\Omega=[0,1]$ and the case $d=2$ where $X=\left(X_{1}, X_{2}\right)$ is uniformly distributed on $\Omega=[0,1]^{2}$.

- Let us first focus on the case $d=1$. Three frontiers are considered:

$$
\begin{aligned}
& g_{1}(x)= \begin{cases}1+\exp \left(-60(x-1 / 4)^{2}\right) & \text { if } x \in[0,1 / 3], \\
1+\exp (-5 / 12) & \text { if } x \in] 1 / 3,2 / 3], \\
1+5 \exp (-5 / 12)-6 \exp (-5 / 12) x & \text { if } x \in] 2 / 3,5 / 6], \\
6 x-4 & \text { if } x \in] 5 / 6,1],\end{cases} \\
& g_{2}(x)=\left(\frac{1}{10}+\sin (\pi x)\right)\left[\frac{11}{10}-\frac{1}{2} \exp \left(-64\left(x-\frac{1}{2}\right)^{2}\right)\right] \\
& g_{3}(x)=\frac{5}{4}-2 x(1-x) .
\end{aligned}
$$

Note that $g_{1}$ is continuous but not differentiable at $x=1 / 3, x=2 / 3$ and $x=5 / 6$ while $g_{2}$ and $g_{3}$ are infinitely differentiable.

In the parametric setting $(P)$, two different models for the function $\alpha(x)$ are considered: a constant function $\alpha_{1}(x)=1.25$ and $\alpha_{2}(x)=1.25+0.5|\cos (2 \pi x)|$.

In the nonparametric setting $(N P)$, the simulated model is given by

$$
\bar{F}(y \mid x)=C(x)\left(1-y / g_{3}(x)\right)^{\alpha_{2}(x)}+(1-C(x))\left(1-y / g_{3}(x)\right)^{\alpha_{2}(x)+1 / 4}, \quad \forall y \in\left[0, g_{3}(x)\right],
$$

with $C(x)=c+\sin (2 \pi x) / 16$ and $c \in\{1 / 8,3 / 8,5 / 8,7 / 8\}$. Let us highlight that (13) can be seen as a "contamination" of the parametric model $(P)$ : the smaller $c$ is, the larger the contamination is.

The uniform kernel is chosen:

$$
K(x)=\frac{1}{2} \mathbb{1}_{[-1,1]}(x)
$$

with associated bandwidth $h_{n}^{(m)}=2 \widehat{\sigma}(X) / n^{1 /\left(1+\alpha_{\infty}\right)}$ and $p_{n}^{(m)}=n^{1 /\left(1+\alpha_{\infty}\right)} / \sqrt{\log (n)}$, where $n=500$ is the sample size, $\widehat{\sigma}(X)$ is the empirical standard deviation of $X$ and $\alpha_{\infty}=$ $\max _{\Omega} \alpha<\infty$ since $\alpha$ is continuous and $\Omega$ is a compact subset of $\mathbb{R}$. These sequences are chosen to check the hypotheses of Theorem 1 . Note that the multiplicative constant $\widehat{\sigma}(X)$ has been suggested by Girard and Jacob (2008), whereas the constant 2 was empirically chosen. 
An alternative approach would be the selection of the bandwidth by cross-validation. Härdle and Marron (1985) have shown that this method is asymptotically optimal for the regression function estimation. Establishing a similar result for the estimation of conditional moments of high order is an open problem.

- In the case $d=2$, we limit ourselves to a unique model

$$
g(x, y)=1+3 g_{1}(x) y / 20, \text { and } \alpha(x, y)=1.25+0.5|\cos (2 \pi x) \sin (2 \pi y)|,
$$

the kernel being

$$
K(x, y)=\frac{1}{4} \mathbb{1}_{[-1,1] \times[-1,1]}(x, y),
$$

with bandwidth $h_{n}^{(m)}=4 \sqrt{\widehat{\sigma}\left(X_{1}\right) \widehat{\sigma}\left(X_{2}\right)} / n^{1 /\left(2+\alpha_{\infty}\right)}$ and $p_{n}^{(m)}=n^{1 /\left(2+\alpha_{\infty}\right)} / \sqrt{\log (n)}$. The sample size is fixed to $n=1000$.

Our estimator is compared to the two estimators proposed by Girard and Jacob (2008) and Geffroy (1964). Let us recall that, similarly to $\widehat{g}_{n}(x)$, Girard and Jacob's estimator (2008) is based on a kernel regression on high power transformed data. At the opposite, the estimator in Geffroy (1964) is based on the extreme values of the sample and does not involve any smoothing. For Girard and Jacob's estimator, we set $h_{n}^{(g j)}=4 \widehat{\sigma}(X) / \sqrt{n}$ and $p_{n}^{(g j)}=\sqrt{n / \log (n)}$ if $d=1$, and $h_{n}^{(g j)}=4 \sqrt{\widehat{\sigma}\left(X_{1}\right) \widehat{\sigma}\left(X_{2}\right)} / n^{1 / 3}$ and $p_{n}^{(g j)}=n^{1 / 3} / \sqrt{\log (n)}$ when $d=2$. The $L^{1}$-errors associated to each estimator are computed on 500 replications of the initial sample of size $n$ and the minimum, maximum and mean $L^{1}$-errors are reported in Table 1. Note that for the moment estimator, these results were obtained with $a=15$, the constant $a$ having been chosen after intensive simulations.

It appears that, in all the considered situations, our moment estimator yields better results than both the estimators of Girard and Jacob (2008) and Geffroy (1964). For a fixed frontier, all the estimators perform better on the situation $\alpha(x)=\alpha_{1}(x)$ than on the situation $\alpha(x)=$ $\alpha_{2}(x)$. This behavior is a consequence of $\alpha_{2}(x)>\alpha_{1}(x)$ : as $\alpha(x)$ increases, the simulated points tend to move away from the frontier $g(x)$. This phenomenon is illustrated in the case $d=1$ on Figures 2 and 3. On each of the upper panels the best situation is represented, i.e. the replication that yields the smallest $L^{1}$-error for $\widehat{g}_{n}$ in Table 1 . Similarly, the worst situation is depicted on the lower panels, i.e. the replication that yields the largest $L^{1}$-error for $\widehat{g}_{n}$ in Table 1. In all cases, $\widehat{g}_{n}$ is superimposed to the frontier $g$. Finally, we show on Figure 4 some $95 \%$ pointwise confidence intervals obtained thanks to Corollary 1 for the frontier $g_{1}$ with $\alpha(x)=\alpha_{2}(x)$ and a parameter $a=3$, in both the best situation (top panel) and the worst situation (bottom panel). These intervals are globally satisfactory.

When $d=2$, scatter plots $\left(g\left(X_{i}\right), \widehat{g}\left(X_{i}\right)\right), i=1, \ldots, n$ are represented on Figure $5, \widehat{g}$ being either our moment estimator or Girard and Jacob's estimator. The best and worst situations are depicted for these two estimators. It appears that the points associated to the moment 
estimator are closer to the line $y=x$ than the points associated to Girard and Jacob's estimator.

\section{Acknowledgements}

The authors are indebted to the anonymous referees for their helpful comments and suggestions that have contributed to an improved presentation of the results of this paper.

\section{References}

Abramovitz, M., Stegun, I. (1965). Handbook of Mathematical Functions, Dover.

Aigner, D.J., Amemiya, T., Poirier, D.J. (1976). On the estimation of production frontier: maximum likelihood estimation on the parameters of a discontinuous density function. International Economic Review, 17:377-396.

Aragon, Y., Daouia, A., Thomas-Agnan, C. (2005). Nonparametric frontier estimation: a conditional quantile-based approach. Journal of Econometric Theory, 21(2):358-389.

Billingsley, P. (1979). Probability and measure, John Wiley and Sons.

Cazals, C., Florens, J.-P., Simar, L. (2002). Nonparametric frontier estimation: A robust approach. Journal of Econometrics, 106(1):1-25.

Chow, Y.S., Teicher, H. (1997). Probability Theory, Springer.

Daouia, A., Florens, J.-P., Simar, L. (2010). Frontier estimation and extreme value theory. Bernoulli, 16(4):1039-1063.

Daouia, A., Florens, J.-P., Simar, L. (2012). Regularization of non parametric frontier estimators. Journal of Econometrics, 168(2):285-299.

Daouia, A., Simar, L. (2005). Robust nonparametric estimators of monotone boundaries. Journal of Multivariate Analysis, 96:311-331.

Deprins, D., Simar, L., Tulkens, H. (1984). Measuring labor efficiency in post offices. In P. Pestieau, M. Marchand and H. Tulkens, editors, The Performance of Public Enterprises: Concepts and Measurements. North Holland ed, Amsterdam.

Embrechts, P., Klüppelberg, C., Mikosch, T. (1997). Modelling extremal events, Springer.

Fan, Y., Li, Q., Weersink, A. (1996). Semiparametric estimation of stochastic production frontier models. Journal of Business and Economic Statistics, 14:460-468.

Farrell, M.J. (1957). The measurement of productive efficiency. Journal of the Royal Statistical Society A, 120:253-281. 
Ferraty, F., Vieu, P. (2005). Nonparametric modelling for functional data. Springer.

Gardes, L. (2002). Estimating the support of a Poisson process via the Faber-Shauder basis and extreme values. Publications de l'Institut de Statistique de l'Université de Paris, XXXXVI:43-72.

Geffroy, J. (1964). Sur un problème d'estimation géométrique. Publications de l'Institut de Statistique de l'Université de Paris, XIII:191-210.

Gijbels, I., Mammen, E., Park, B.U., Simar, L. (1999). On estimation of monotone and concave frontier functions. Journal of the American Statistical Association, 94(445):220228.

Girard, S., Iouditski, A., Nazin, A. (2005). $L_{1}$-optimal nonparametric frontier estimation via linear programming. Automation and Remote Control, 66(12):2000-2018.

Girard, S., Jacob, P. (2003a). Extreme values and Haar series estimates of point process boundaries. Scandinavian Journal of Statistics, 30(2):369-384.

Girard, S., Jacob, P. (2003b). Projection estimates of point processes boundaries. Journal of Statistical Planning and Inference, 116(1):1-15.

Girard, S., Jacob, P. (2004). Extreme values and kernel estimates of point processes boundaries. ESAIM: Probability and Statistics, 8:150-168.

Girard, S., Jacob, P. (2008). Frontier estimation via kernel regression on high power transformed data. Journal of Multivariate Analysis, 99:403-420.

Girard, S., Menneteau, L. (2005). Central limit theorems for smoothed extreme value estimates of point processes boundaries. Journal of Statistical Planning and Inference, 135(2):433-460.

Hall, P., Park, B.U. (2004). Bandwidth choice for local polynomial estimation of smooth boundaries. Journal of Multivariate Analysis, 91(2):240-261.

Hall, P., Park, B.U., Stern, S.E. (1998). On polynomial estimators of frontiers and boundaries. Journal of Multivariate Analysis, 66(1):71-98.

Härdle, W., Marron, J.S. (1985). Optimal bandwidth selection in nonparametric regression function estimation. The Annals of Statistics, 13(4):1465-1481.

Härdle, W., Park, B.U., Tsybakov, A. (1995). Estimation of non-sharp support boundaries. Journal of Multivariate Analysis, 43:205-218.

Härdle, W., Stoker, T.M. (1989). Investigating smooth multiple regression by the method of average derivatives. Journal of the American Statistical Association, 84:986-995.

Jacob, P., Suquet, C. (1995). Estimating the edge of a Poisson process by orthogonal series. 
Journal of Statistical Planning and Inference, 46:215-234.

Knight, K. (2001). Limiting distributions of linear programming estimators. Extremes, 4(2):87-103.

Korostelev, A., Simar, L., Tsybakov, A. (1995). Efficient estimation of monotone boundaries. The Annals of Statistics, 23:476-489.

Korostelev, A., Tsybakov, A. (1993). Minimax theory of image reconstruction, volume 82 of Lecture Notes in Statistics. Springer-Verlag, New-York.

Kumbhakar, S.C., Park, A., Simar, S., Tsionas, E. (2007). Nonparametric stochastic frontiers: A local maximum likelihood approach. Journal of Econometrics, 237:1-27.

Menneteau, L. (2008). Multidimensional limit theorems for smoothed extreme value estimates of point processes boundaries. ESAIM: Probability and Statistics, 12:273-307.

Parzen, E. (1962). On estimation of a probability density function and mode. Annals of Mathematical Statistics, 33(3):1065-1076.

Simar, L., Zelenyuk, V. (2011). Stochastic FDH/DEA estimators for frontier analysis. Journal of Productivity Analysis, 36:1-20.

Tricomi, F.G., Erdélyi, A. (1951). The asymptotic expansion of a ratio of gamma functions, Pacific Journal of Mathematics, 1:133-142.

\section{Appendix A: Auxiliary results}

First, some results on the moments $\mu_{p_{n}}(x)$ and $m_{1, p_{n}}(x)$ are provided, see (4) for a definition.

Lemma 1. Suppose that $(N P),(K)$ and $\left(A_{1}-A_{3}\right)$ hold. Let $x \in \Omega$ such that $f(x)>0$. If $p_{n} h_{n}^{\eta_{g}} \rightarrow 0$, then

i) $\mu_{p_{n}}(x)=f(x) g^{p_{n}}(x) m_{1, p_{n}}(x)(1+\mathrm{o}(1))$.

ii) $m_{1, p_{n}}(x)=m_{1, p_{n}+1}(x)(1+\mathrm{o}(1))$.

The next result of this section is technical: it provides precise expansions of the smoothed moment $\mathbb{E}\left(Y^{p_{n}} K_{h_{n}}^{q}(X-x)\right)$ when $p_{n} \rightarrow \infty, h_{n} \rightarrow 0$ and for all $q \geq 1$. It will be useful for the proof of our next lemmas and of Theorem 2.

Lemma 2. Suppose $(P),(K)$ and $\left(A_{2}-A_{4}\right)$ hold. For all $q \geq 1, u \in B, n \in \mathbb{N} \backslash\{0\}$ and $x \in \Omega$ such that $f(x)>0$, let

$$
\begin{aligned}
L_{n}\left(p_{n}, x, u\right) & =\frac{f\left(x-h_{n} u\right) \Gamma\left(\alpha\left(x-h_{n} u\right)+1\right)}{f(x) \Gamma(\alpha(x)+1)} \exp \left[p_{n} \frac{\Delta_{n}^{g}(x, u)}{g(x)}-\log \left(p_{n}\right) \Delta_{n}^{\alpha}(x, u)\right] \\
\Lambda_{n}\left(q, p_{n}, x\right) & =h_{n}^{d(q-1)} \frac{\mathbb{E}\left(Y^{p_{n}} K_{h_{n}}^{q}(X-x)\right)}{f(x) g^{p_{n}}(x)} .
\end{aligned}
$$


If $p_{n} h_{n}^{\eta_{g}} \rightarrow 0$, then

i) $L_{n}\left(p_{n}, x, u\right) \rightarrow 1$ as $n \rightarrow \infty$ uniformly in $u \in B$.

ii) For all $q \geq 1$,

$$
\begin{aligned}
\frac{\Lambda_{n}\left(q, p_{n}, x\right)}{\alpha(x) B\left(p_{n}+1, \alpha(x)\right)} & =\int_{B} L_{n}\left(p_{n}, x, u\right)\left[1-\frac{p_{n}}{2}\left(\frac{\Delta_{n}^{g}(x, u)}{g(x)}\right)^{2}\right] K^{q}(u) d u \\
& -\frac{1}{p_{n}} \int_{B} L_{n}\left(p_{n}, x, u\right) \frac{\Delta_{n}^{\alpha}(x, u)}{2}\left[\alpha\left(x-h_{n} u\right)+\alpha(x)+1\right] K^{q}(u) d u \\
& +\mathrm{O}\left(\frac{h_{n}^{\eta_{g}}}{p_{n}}+\frac{h_{n}^{\eta_{\alpha}}}{p_{n}^{2}}\right) .
\end{aligned}
$$

iii) Moreover, there exist $\delta_{1}, \delta_{2} \in \mathbb{R}$ such that for all $q \geq 1$,

$$
\begin{aligned}
\frac{\Lambda_{n}\left(q, p_{n}, x\right)}{\Gamma(\alpha(x)+1) p_{n}^{-\alpha(x)}} & =\int_{B} L_{n}\left(p_{n}, x, u\right)\left[1+\frac{\delta_{1}}{p_{n}}-\frac{p_{n}}{2}\left(\frac{\Delta_{n}^{g}(x, u)}{g(x)}\right)^{2}\right] K^{q}(u) d u \\
& -\frac{1}{p_{n}} \int_{B} L_{n}\left(p_{n}, x, u\right) \frac{\Delta_{n}^{\alpha}(x, u)}{2}\left[\alpha\left(x-h_{n} u\right)+\alpha(x)+1\right] K^{q}(u) d u \\
& +\frac{\delta_{2}}{p_{n}^{2}} \int_{B} K^{q}(u) d u+\mathrm{o}\left(p_{n}^{-2}\right) .
\end{aligned}
$$

Our next lemma consists in linearizing

$$
\xi_{n}(x)=\frac{g(x)}{\|K\|_{2}} \sqrt{\frac{f(x)}{V(\alpha(x), a)}} \cdot v_{n}(x)\left(\frac{1}{\widehat{g}_{n}(x)}-\frac{1}{G_{n}(x)}\right)
$$

appearing in Theorem 2 .

Lemma 3. Suppose $(P),(K),\left(A_{2}-A_{4}\right)$ hold and let $x \in \Omega$ such that $f(x)>0$. If $p_{n} \rightarrow \infty$ then

$$
\begin{aligned}
\xi_{n}(x) & =\left[\zeta_{n}^{(1)}(x)+\left(\frac{\mu_{p_{n}+1}(x)}{\widehat{\mu}_{p_{n}+1}(x)}-1\right) \zeta_{n}^{(2)}(x)+\left(1+\frac{a p_{n}}{p_{n}+1}\right)\left(\frac{\mu_{(a+1) p_{n}+1}(x)}{\widehat{\mu}_{(a+1) p_{n}+1}(x)}-1\right) \zeta_{n}^{(3)}(x)\right] \\
& \times u_{n, a}(x)(1+\mathrm{o}(1))
\end{aligned}
$$

where $\nu_{p}(x)=\widehat{\mu}_{p}(x)-\mu_{p}(x)$,

$$
\begin{aligned}
\zeta_{n}^{(1)}(x) & =\zeta_{n}^{(2)}(x)+\left[1+\frac{a p_{n}}{p_{n}+1}\right] \zeta_{n}^{(3)}(x) \\
\text { with } \zeta_{n}^{(2)}(x) & =-\nu_{p_{n}}(x)+\frac{\mu_{p_{n}}(x)}{\mu_{p_{n}+1}(x)} \nu_{p_{n}+1}(x) \\
\zeta_{n}^{(3)}(x) & =\frac{\mu_{p_{n}+1}(x)}{\mu_{(a+1) p_{n}+1}(x)} \nu_{(a+1) p_{n}}(x)-\frac{\mu_{p_{n}+1}(x) \mu_{(a+1) p_{n}}(x)}{\mu_{(a+1) p_{n}+1}^{2}(x)} \nu_{(a+1) p_{n}+1}(x) \\
\text { and } u_{n, a}(x) & =\frac{1}{a\|K\|_{2} \Gamma(\alpha(x)+1)} \sqrt{\frac{1}{f(x) V(\alpha(x), a)}} \frac{p_{n}^{\alpha(x)} v_{n}(x)}{g^{p_{n}}(x)}
\end{aligned}
$$

Finally, the following result provides an asymptotic bound of the third-order moments appearing in the proofs.

Lemma 4. Suppose $(P),(K),\left(A_{2}-A_{4}\right)$ are satisfied and $p_{n} h_{n}^{\eta_{g}} \rightarrow 0$ as $n \rightarrow \infty$. Let $k \in \mathbb{N}$, $\left(b_{n, j}\right)_{n \in \mathbb{N} \backslash\{0\}, 0 \leq j \leq k} \in \mathbb{R}$ and $x \in \mathbb{R}^{d}$ such that there exist $m \in \mathbb{N}$ and sequences of measurable 
functions $\left(H_{n, j}\right), 0 \leq j \leq m$, uniformly bounded on $[0,1]$ with

$$
\forall y \in[0,1] \quad \max _{u \in B}\left|\sum_{j=0}^{k} b_{n, j} g^{j}\left(x-h_{n} u\right) y^{j}\right| \leq \sum_{j=0}^{m} \frac{H_{n, j}(y)}{p_{n}^{j}}(1-y)^{m-j} .
$$

Let us consider

$$
S_{n}(x)=\frac{1}{n} \sum_{j=0}^{k} b_{n, j} Y^{p_{n}+j} K_{h_{n}}\left(x-X_{j}\right)
$$

Then $\mathbb{E}\left|S_{n}(x)\right|^{3}=\mathrm{O}\left(n^{-3} g^{3 p_{n}}(x) p_{n}^{-\alpha(x)-3 m} h_{n}^{-2 d}\right)$.

\section{Appendix B: Proof of the auxiliary results}

Proof of Lemma 1. i) Recall that

$$
\mu_{p_{n}}(x)=\int_{B} f\left(x-h_{n} u\right) g^{p_{n}}\left(x-h_{n} u\right) m_{1, p_{n}}\left(x-h_{n} u\right) K(u) d u .
$$

First, $\left(A_{2}\right)$ yields

$$
\sup _{u \in B}\left|\frac{f\left(x-h_{n} u\right)}{f(x)}-1\right|=\sup _{u \in B}\left|\frac{\Delta_{n}^{f}(x, u)}{f(x)}\right| \leq \frac{\varepsilon_{f} h_{n}^{\eta_{f}}}{f(x)} \rightarrow 0 .
$$

Second, $\left(A_{3}\right)$ entails

$$
p_{n} \sup _{u \in B}\left|\frac{\Delta_{n}^{g}(x, u)}{g(x)}\right| \leq p_{n} \sup _{u \in B}\left\{\frac{\varepsilon_{g} h_{n}^{\eta_{g}}\|u\|^{\eta_{g}}}{g(x)}\right\}=\mathrm{O}\left(p_{n} h_{n}^{\eta_{g}}\right)
$$

so that the hypothesis $p_{n} h_{n}^{\eta_{g}} \rightarrow 0$ gives

$$
\log \left[\frac{g^{p_{n}}\left(x-h_{n} u\right)}{g^{p_{n}}(x)}\right]=p_{n} \log \left[1+\frac{\Delta_{n}^{g}(x, u)}{g(x)}\right]=\mathrm{O}\left(p_{n} h_{n}^{\eta_{g}}\right) \rightarrow 0
$$

uniformly in $u \in B$ as $n \rightarrow \infty$. Finally, setting

$$
I_{p_{n}}(x):=\int_{0}^{1} y^{p_{n}-1} \overline{F_{1}}(y \mid x) d y
$$

one has $m_{1, p_{n}}(x)=p_{n} I_{p_{n}}(x)$, and $\left(A_{1}\right)$ yields

$$
\sup _{u \in B}\left|\frac{I_{p_{n}}\left(x-h_{n} u\right)}{I_{p_{n}}(x)}-1\right| \rightarrow 0
$$

as $n \rightarrow \infty$. Collecting (14), (16) and (17), the dominated convergence theorem therefore gives i).

ii) Pick $\varepsilon>0$. The integral $I_{p_{n}}(x)$ is rewritten as:

$$
I_{p_{n}}(x)=\int_{1-\varepsilon}^{1} y^{p_{n}-1} \overline{F_{1}}(y \mid x) d y\left[1+\frac{\int_{0}^{1-\varepsilon} y^{p_{n}-1} \overline{F_{1}}(y \mid x) d y}{\int_{1-\varepsilon}^{1} y^{p_{n}-1} \overline{F_{1}}(y \mid x) d y}\right] ;
$$


with

$$
\begin{aligned}
0 \leq \frac{\int_{0}^{1-\varepsilon} y^{p_{n}-1} \overline{F_{1}}(y \mid x) d y}{\int_{1-\varepsilon}^{1} y^{p_{n}-1} \overline{F_{1}}(y \mid x) d y} & \leq \frac{1-\varepsilon}{\int_{1-\varepsilon}^{1}\left[\frac{y}{1-\varepsilon}\right]^{p_{n}-1} \overline{F_{1}}(y \mid x) d y} \\
& \leq \frac{1-\varepsilon}{\left[\frac{1-\varepsilon / 2}{1-\varepsilon}\right]^{p_{n}-1} \int_{1-\varepsilon / 2}^{1} \overline{F_{1}}(y \mid x) d y} .
\end{aligned}
$$

Because $\left[\frac{1-\varepsilon / 2}{1-\varepsilon}\right]^{p_{n}-1} \rightarrow \infty$ as $n \rightarrow \infty$, we therefore get:

$$
I_{p_{n}}(x)=\int_{1-\varepsilon}^{1} y^{p_{n}-1} \overline{F_{1}}(y \mid x) d y(1+\mathrm{o}(1)) .
$$

Since

$$
1 \leq \int_{1-\varepsilon}^{1} y^{p_{n}-1} \overline{F_{1}}(y \mid x) d y / \int_{1-\varepsilon}^{1} y^{p_{n}} \overline{F_{1}}(y \mid x) d y \leq \frac{1}{1-\varepsilon}
$$

for all $\varepsilon>0$, one has $I_{p_{n}}(x) / I_{p_{n}+1}(x) \rightarrow 1$ as $n \rightarrow \infty$. Hence, $m_{1, p_{n}}(x) / m_{1, p_{n}+1}(x) \rightarrow 1$ as $n \rightarrow \infty$, which completes the proof of ii).

Proof of Lemma 2. i) Let us introduce

$$
Q_{n}(x, u)=\frac{f\left(x-h_{n} u\right) \Gamma\left(\alpha\left(x-h_{n} u\right)+1\right)}{f(x) \Gamma(\alpha(x)+1)} .
$$

Since $f$ and $\alpha$ are continuous at $x$ and $\Gamma$ is continuous on $(0, \infty)$, one has $Q_{n}(x, u) \rightarrow 1$ as $n \rightarrow \infty$, uniformly in $u \in \mathcal{B}$. Moreover, since $h_{n}^{\eta_{g}} p_{n} \rightarrow 0$, we have

$$
\sup _{u \in B} \log \left(p_{n}\right)\left|\Delta_{n}^{\alpha}(x, u)\right| \leq \varepsilon_{\alpha} h_{n}^{\eta_{\alpha}}\left|\log p_{n}\right|=\varepsilon_{\alpha}\left[h_{n}^{\eta_{g}} p_{n}\right]^{\eta_{\alpha} / \eta_{g}} \frac{\left|\log p_{n}\right|}{p_{n}^{\eta_{\alpha} / \eta_{g}}} \rightarrow 0 .
$$

It was already proved that $\sup _{u \in B} p_{n}\left|\Delta_{n}^{g}(x, u)\right| \rightarrow 0$ as $n \rightarrow \infty$, see (15). As a conclusion, $L_{n}\left(p_{n}, x, u\right) \rightarrow 1$ as $n \rightarrow \infty$, uniformly in $u \in B$.

ii) By definition of the Beta function,

$$
\frac{\Lambda_{n}\left(q, p_{n}, x\right)}{\alpha(x) B\left(p_{n}+1, \alpha(x)\right)}=\int_{B} Q_{n}(x, u) \frac{\Gamma\left(p_{n}+1+\alpha(x)\right)}{\Gamma\left(p_{n}+1+\alpha\left(x-h_{n} u\right)\right)} \frac{g^{p_{n}}\left(x-h_{n} u\right)}{g^{p_{n}}(x)} K^{q}(u) d u .
$$

Recall now that for all $z>0$, one has

$$
\log \Gamma(z)=\left(z-\frac{1}{2}\right) \log z-z+\frac{\log 2 \pi}{2}+2 \int_{0}^{\infty} \frac{\arctan (t / z)}{e^{2 \pi t}-1} d t
$$

(see Formula 6.1.50 p. 258 in Abramovitz and Stegun, 1965). Using the mean value theorem, simple calculations then yield

$$
\begin{aligned}
\frac{\Gamma\left(p_{n}+1+\alpha(x)\right)}{\Gamma\left(p_{n}+1+\alpha\left(x-h_{n} u\right)\right)} & =\exp \left(-\log \left(p_{n}\right) \Delta_{n}^{\alpha}(x, u)\right)\left(1-\frac{\Delta_{n}^{\alpha}(x, u)}{2 p_{n}}\left(1+\alpha\left(x-h_{n} u\right)+\alpha(x)\right)\right) \\
& +\mathrm{O}\left(\frac{h_{n}^{\eta_{\alpha}}}{p_{n}^{2}}\right),
\end{aligned}
$$


uniformly in $u \in B$. Besides

$$
\begin{aligned}
\frac{g^{p_{n}}\left(x-h_{n} u\right)}{g^{p_{n}}(x)} & =\exp \left[p_{n} \log \left(1+\frac{\Delta_{n}^{g}(x, u)}{g(x)}\right)\right] \\
& =\exp \left[p_{n} \frac{\Delta_{n}^{g}(x, u)}{g(x)}\right]\left[1-\frac{p_{n}}{2}\left(\frac{\Delta_{n}^{g}(x, u)}{g(x)}\right)^{2}\right]+\mathrm{O}\left(\frac{h_{n}^{\eta_{g}}}{p_{n}}\right)
\end{aligned}
$$

uniformly in $u \in B$. Replacing (19) and (20) in (18) gives the first desired expansion.

iii) Now, according to Tricomi and Erdélyi (1951), for all $\kappa$ and $\iota$, there exist two real numbers $\delta_{1}(\kappa, \iota)$ and $\delta_{2}(\kappa, \iota)$ such that

$$
\frac{\Gamma(x+\kappa)}{\Gamma(x+\iota)}=x^{\kappa-\iota}\left[1+\frac{\delta_{1}(\kappa, \iota)}{x}+\frac{\delta_{2}(\kappa, \iota)}{x^{2}}+\mathrm{o}\left(\frac{1}{x^{2}}\right)\right] .
$$

Consequently, setting $\delta_{1}=\delta_{1}(1, \alpha(x)+1)$ and $\delta_{2}=\delta_{2}(1, \alpha(x)+1)$, we have

$$
B\left(p_{n}+1, \alpha(x)\right)=\Gamma(\alpha(x)) p_{n}^{-\alpha(x)}\left[1+\frac{\delta_{1}}{p_{n}}+\frac{\delta_{2}}{p_{n}^{2}}+\mathrm{o}\left(\frac{1}{p_{n}^{2}}\right)\right] .
$$

Replacing in the expansion ii) and remarking that, from i),

$$
\int_{B} L_{n}\left(p_{n}, x, u\right) \frac{\delta_{2}}{p_{n}^{2}} K^{q}(u) d u=\frac{\delta_{2}}{p_{n}^{2}} \int_{B} K^{q}(u) d u+\mathrm{o}\left(\frac{1}{p_{n}^{2}}\right)
$$

yields iii).

Proof of Lemma 3. Let us first remark that, from Lemma 2i) and iii) with $q=1$,

$$
\mu_{p_{n}+1}(x)=f(x) \Gamma(\alpha(x)+1) g^{p_{n}+1}(x) p_{n}^{-\alpha(x)}(1+\mathrm{o}(1)),
$$

leading to

$$
\mu_{p_{n}+1}(x) u_{n, a}(x)=\frac{g(x)}{a\|K\|_{2}} \sqrt{\frac{f(x)}{V(\alpha(x), a)}} \cdot v_{n}(x)(1+\mathrm{o}(1)),
$$

and therefore

$$
\xi_{n}(x)=\frac{\mu_{p_{n}+1}(x) u_{n, a}(x)}{p_{n}+1} \cdot a p_{n}\left(\frac{1}{\widehat{g}_{n}(x)}-\frac{1}{G_{n}(x)}\right)(1+\mathrm{o}(1)) .
$$

Besides,

$$
\begin{aligned}
a p_{n}\left(\frac{1}{\widehat{g}_{n}(x)}-\frac{1}{G_{n}(x)}\right) & =\left((a+1) p_{n}+1\right) \frac{\widehat{\mu}_{(a+1) p_{n}}(x) \mu_{(a+1) p_{n}+1}(x)-\mu_{(a+1) p_{n}}(x) \widehat{\mu}_{(a+1) p_{n}+1}(x)}{\widehat{\mu}_{(a+1) p_{n}+1}(x) \mu_{(a+1) p_{n}+1}(x)} \\
& -\left(p_{n}+1\right) \frac{\widehat{\mu}_{p_{n}}(x) \mu_{p_{n}+1}(x)-\mu_{p_{n}}(x) \widehat{\mu}_{p_{n}+1}(x)}{\widehat{\mu}_{p_{n}+1}(x) \mu_{p_{n}+1}(x)} \\
=: & D_{n}^{(1)}(x)-D_{n}^{(2)}(x)
\end{aligned}
$$

with

$$
\begin{aligned}
D_{n}^{(1)}(x) & :=\frac{(a+1) p_{n}+1}{\mu_{(a+1) p_{n}+1}(x)} \cdot \frac{\mu_{(a+1) p_{n}+1}(x)}{\widehat{\mu}_{(a+1) p_{n}+1}(x)} \cdot\left(\nu_{(a+1) p_{n}}(x)-\frac{\mu_{(a+1) p_{n}}(x)}{\mu_{(a+1) p_{n}+1}(x)} \nu_{(a+1) p_{n}+1}(x)\right) \\
D_{n}^{(2)}(x) & :=\frac{p_{n}+1}{\mu_{p_{n}+1}(x)} \cdot \frac{\mu_{p_{n}+1}(x)}{\widehat{\mu}_{p_{n}+1}(x)} \cdot\left(\nu_{p_{n}}(x)-\frac{\mu_{p_{n}}(x)}{\mu_{p_{n}+1}(x)} \nu_{p_{n}+1}(x)\right)
\end{aligned}
$$


which leads to

$$
\begin{aligned}
& \frac{\mu_{p_{n}+1}(x)}{p_{n}+1} \cdot D_{n}^{(1)}(x)=\left(1+\frac{a p_{n}}{p_{n}+1}\right) \frac{\mu_{(a+1) p_{n}+1}(x)}{\widehat{\mu}_{(a+1) p_{n}+1}(x)} \cdot \zeta_{n}^{(3)}(x) \\
& \frac{\mu_{p_{n}+1}(x)}{p_{n}+1} \cdot D_{n}^{(2)}(x)=-\frac{\mu_{p_{n}+1}(x)}{\widehat{\mu}_{p_{n}+1}(x)} \cdot \zeta_{n}^{(2)}(x) .
\end{aligned}
$$

Replacing in (22) concludes the proof of Lemma 3.

Proof of Lemma 4. Conditioning on $X$ yields

$$
\begin{aligned}
\mathbb{E}\left|S_{n}(x)\right|^{3} & =\frac{1}{n^{3}} \int_{\mathbb{R}^{d}} \mathbb{E}\left[\left|\sum_{j=0}^{k} b_{n, j} Y^{p_{n}+j} K_{h_{n}}(x-v)\right|^{3} \mid X=v\right] f(v) d v \\
& =\frac{1}{n^{3} h_{n}^{2 d}} \int_{B} \mathbb{E}\left[\left|\sum_{j=0}^{k} b_{n, j} Y^{p_{n}+j}\right|^{3} \mid X=x-h_{n} u\right] K^{3}(u) f\left(x-h_{n} u\right) d u .
\end{aligned}
$$

Now, given $\left\{X=x-h_{n} u\right\}$, we have $W_{n}(x, u):=\frac{Y}{g\left(x-h_{n} u\right)} \leq 1$. Setting

$$
c_{n}(x):=(m+1)^{2} \sup _{\substack{[0,1] \\ 0 \leq j \leq m \\ n \in \mathbb{N} \backslash\{0\}}}\left|H_{n, j}\right|^{3} \cdot \max _{u \in B} \frac{g^{3 p_{n}}\left(x-h_{n} u\right)}{g^{3 p_{n}}(x)},
$$

which is a bounded sequence, Hölder's inequality entails, given $\left\{X=x-h_{n} u\right\}$,

$$
\begin{aligned}
\left|\sum_{j=0}^{k} b_{n, j} Y^{p_{n}+j}\right|^{3} & =g^{3 p_{n}}\left(x-h_{n} u\right)\left|W_{n}^{p_{n}}(x, u) \sum_{j=0}^{k} b_{n, j} W_{n}^{j}(x, u) g^{j}\left(x-h_{n} u\right)\right|^{3} \\
& \leq c_{n}(x) g^{3 p_{n}}(x) \sum_{j=0}^{m} \frac{1}{p_{n}^{3 j}} W_{n}^{3 p_{n}}(x, u)\left(1-W_{n}(x, u)\right)^{3(m-j)} .
\end{aligned}
$$

It is therefore sufficient to prove that, for all $j \in\{0, \ldots, m\}$, uniformly in $u \in B$,

$$
\mathbb{E}\left(W_{n}^{3 p_{n}}(x, u)\left(1-W_{n}(x, u)\right)^{3(m-j)} \mid X=x-h_{n} u\right)=\mathrm{O}\left(p_{n}^{-\alpha(x)-(3 m-3 j)}\right) .
$$

Because for all $\lambda, \mu \geq 0$, the function

$$
(y, \omega) \mapsto \frac{d}{d y}\left[y^{\lambda}(1-y)^{\mu}\right] \mathbb{1}_{\left\{y \leq W_{n}(x, u)(\omega)\right\}}
$$

is Lebesgue $\otimes \mathbb{P}\left(\cdot \mid X=x-h_{n} u\right)$-integrable, Fubini's theorem gives

$\mathbb{E}\left(W_{n}^{3 p_{n}}(x, u)\left(1-W_{n}(x, u)\right)^{3(m-j)} \mid X=x-h_{n} u\right)=\int_{0}^{1} \frac{d}{d y}\left[y^{3 p_{n}}(1-y)^{3(m-j)}\right] \bar{F}_{1}\left(y \mid x-h_{n} u\right) d y$ since, given $\left\{X=x-h_{n} u\right\}, W_{n}(x, u)$ has survival function $\bar{F}_{1}\left(\cdot \mid x-h_{n} u\right)$. To conclude, notice that if $\left(s_{n}\right)$ is a real sequence tending to infinity such that $s_{n} h_{n}^{\eta_{g}} \rightarrow 0$ as $n \rightarrow \infty$ and $\ell \geq 0$, we obtain following (19) and Tricomi and Erdélyi (1951)

$$
\int_{0}^{1} y^{s_{n}}(1-y)^{\ell+\alpha\left(x-h_{n} u\right)} d y=B\left(s_{n}+1, \ell+\alpha\left(x-h_{n} u\right)+1\right)=\mathrm{O}\left(s_{n}^{-\alpha(x)-\ell-1}\right)
$$


uniformly in $u \in B$. Since $\bar{F}_{1}\left(y \mid x-h_{n} u\right)=(1-y)^{\alpha\left(x-h_{n} u\right)}$, some quick computations then show that

$$
\mathbb{E}\left(W_{n}^{3 p_{n}}(x, u)\left(1-W_{n}(x, u)\right)^{3(m-j)} \mid X=x-h_{n} u\right)=\mathrm{O}\left(p_{n}^{-\alpha(x)-(3 m-3 j)}\right)
$$

uniformly in $u \in B$, which ends the proof of Lemma 4 . 


\begin{tabular}{|c|c|c|c|}
\hline Situation & Moment estimator & Girard-Jacob estimator & Geffroy estimator \\
\hline Case $d=1$, model $(P)$ & & & \\
\hline$\alpha(x)=\alpha_{1}$ & & & \\
Frontier $g_{1}$ & $0.082[0.051,0.117]$ & $0.089[0.052,0.135]$ & $0.107[0.058,0.168]$ \\
Frontier $g_{2}$ & $0.045[0.032,0.070]$ & $0.047[0.031,0.078]$ & $0.050[0.029,0.089]$ \\
\hline$\alpha(x)=\alpha_{2}(x)$ & & & \\
Frontier $g_{1}$ & $0.109[0.073,0.179]$ & $0.162[0.093,0.241]$ & $0.169[0.087,0.248]$ \\
Frontier $g_{2}$ & $0.064[0.042,0.088]$ & $0.067[0.037,0.099]$ & $0.072[0.041,0.115]$ \\
\hline Case $d=1$, model $(13)$ & & & \\
\hline$c=7 / 8$ & $0.055[0.032,0.101]$ & $0.108[0.070,0.157]$ & $0.107[0.067,0.174]$ \\
$c=5 / 8$ & $0.058[0.032,0.101]$ & $0.116[0.076,0.161]$ & $0.112[0.069,0.154]$ \\
$c=3 / 8$ & $0.063[0.030,0.111]$ & $0.127[0.083,0.171]$ & $0.122[0.062,0.177]$ \\
$c=1 / 8$ & $0.070[0.037,0.136]$ & $0.137[0.086,0.190]$ & $0.131[0.085,0.194]$ \\
\hline Case $d=2$, model $(P)$ & $0.036[0.024,0.058]$ & $0.146[0.105,0.195]$ & $0.176[0.124,0.213]$ \\
\hline
\end{tabular}

Table 1: Mean $L^{1}-$ errors and [minimum, maximum] $L^{1}$-errors associated to the estimators in the different situations.

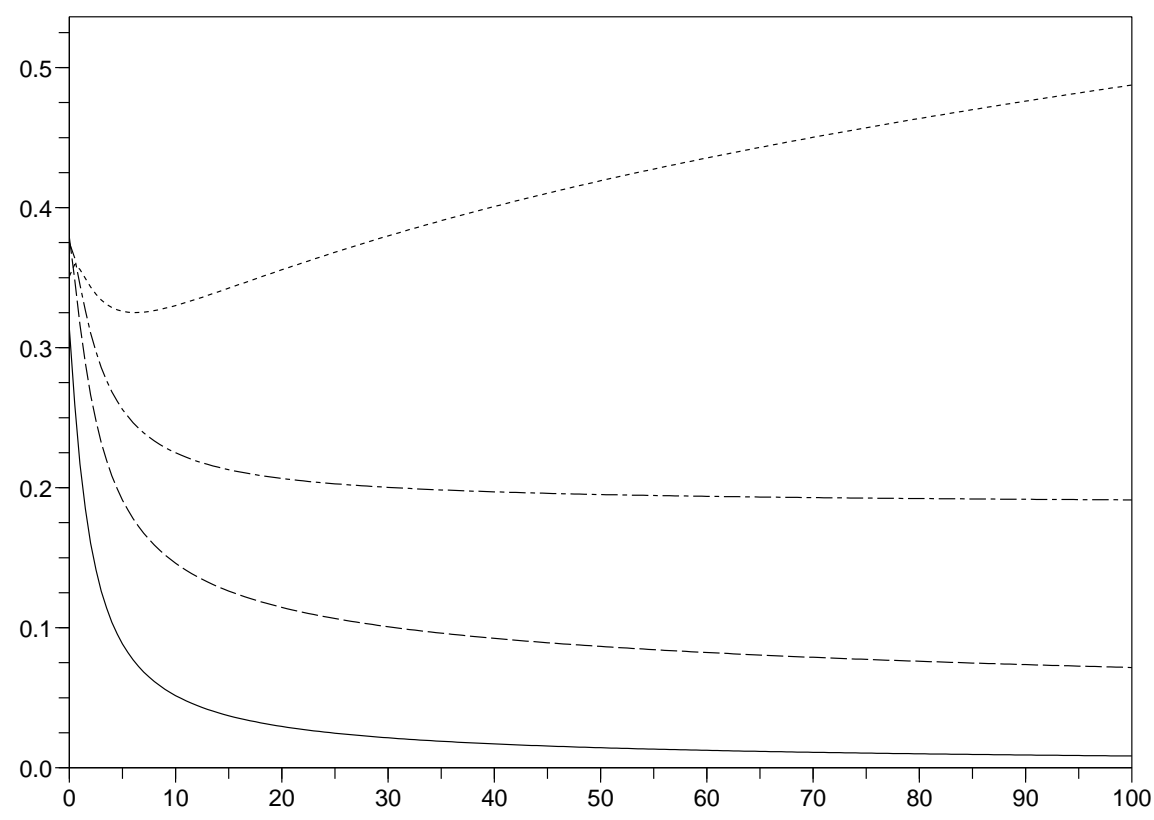

Figure 1: Graphs of the functions $a \mapsto V(\alpha, a)$. Solid line $\alpha=1.25$, dashed line $\alpha=1.75$, dashed-dotted line $\alpha=2$, dotted line $\alpha=2.25$. 

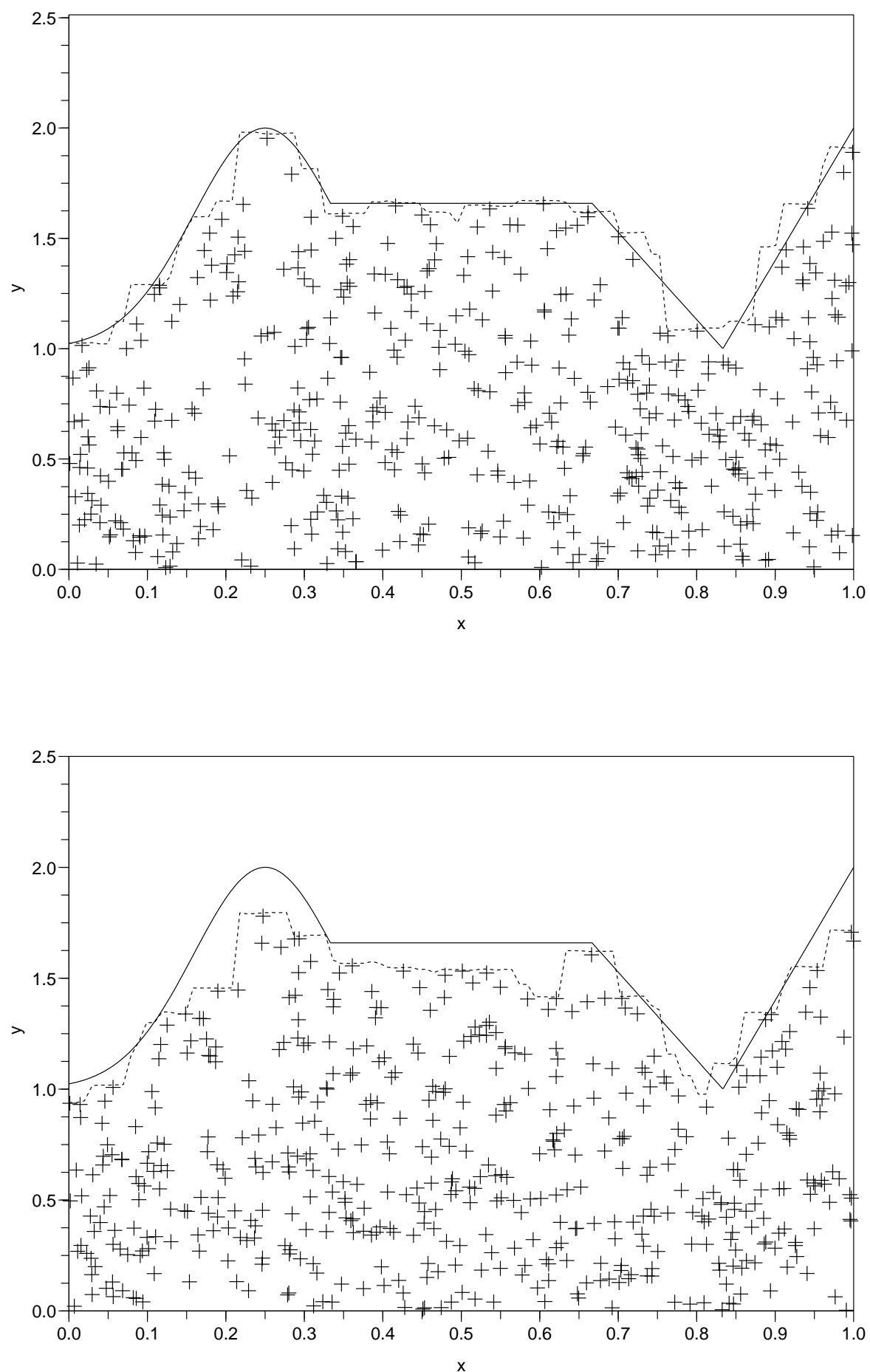

Figure 2: Case $d=1$ and $\alpha(x)=\alpha_{1}$ : the frontier $g_{1}$ (solid line) and its moment estimate $\widehat{g}_{n}$ (dotted line) with $a=15$. Top: best situation, bottom: worst situation. 

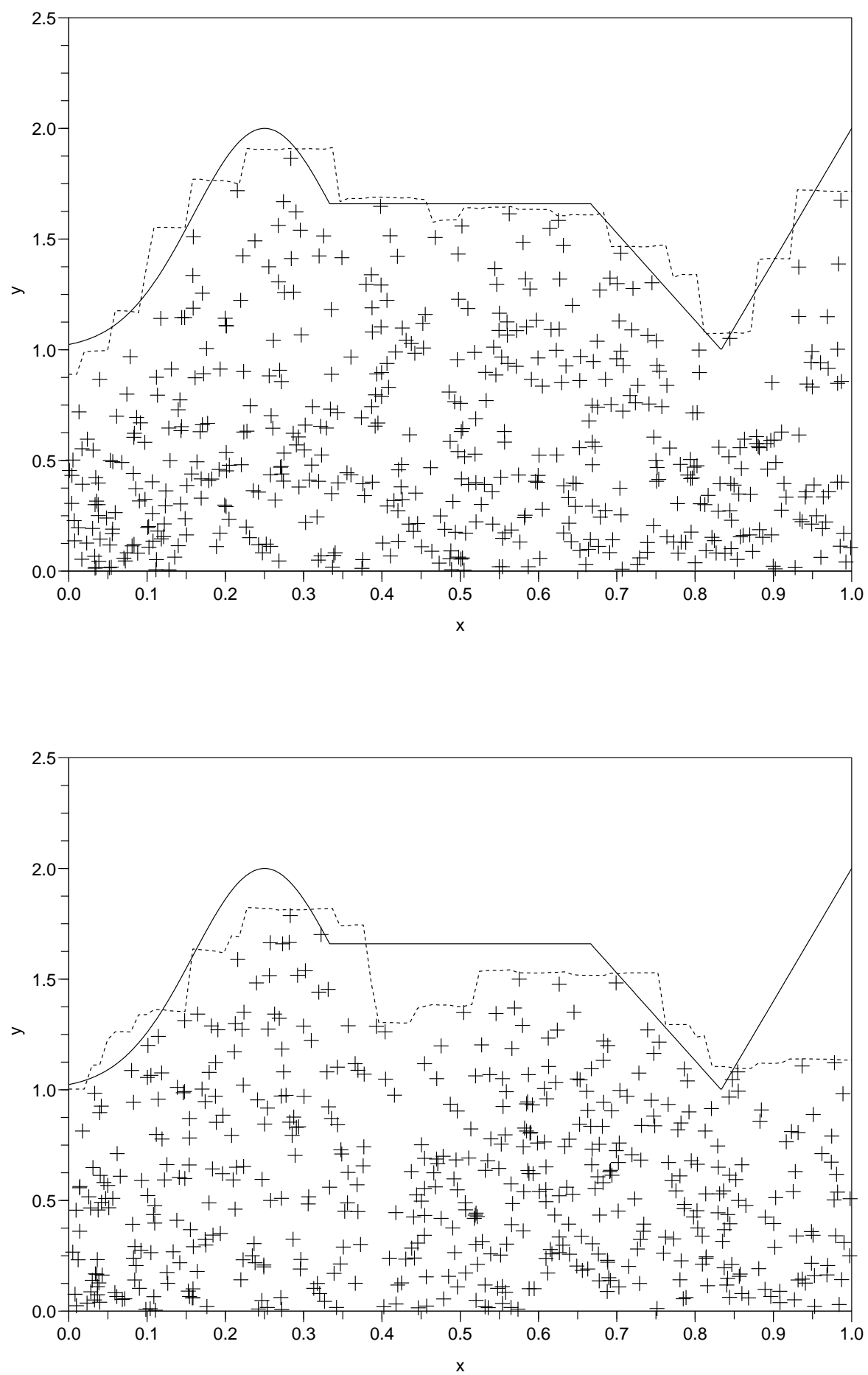

Figure 3: Case $d=1$ and $\alpha(x)=\alpha_{2}(x)$ : the frontier $g_{1}$ (solid line) and its moment estimate $\widehat{g}_{n}$ (dotted line) with $a=15$. Top: best situation, bottom: worst situation. 

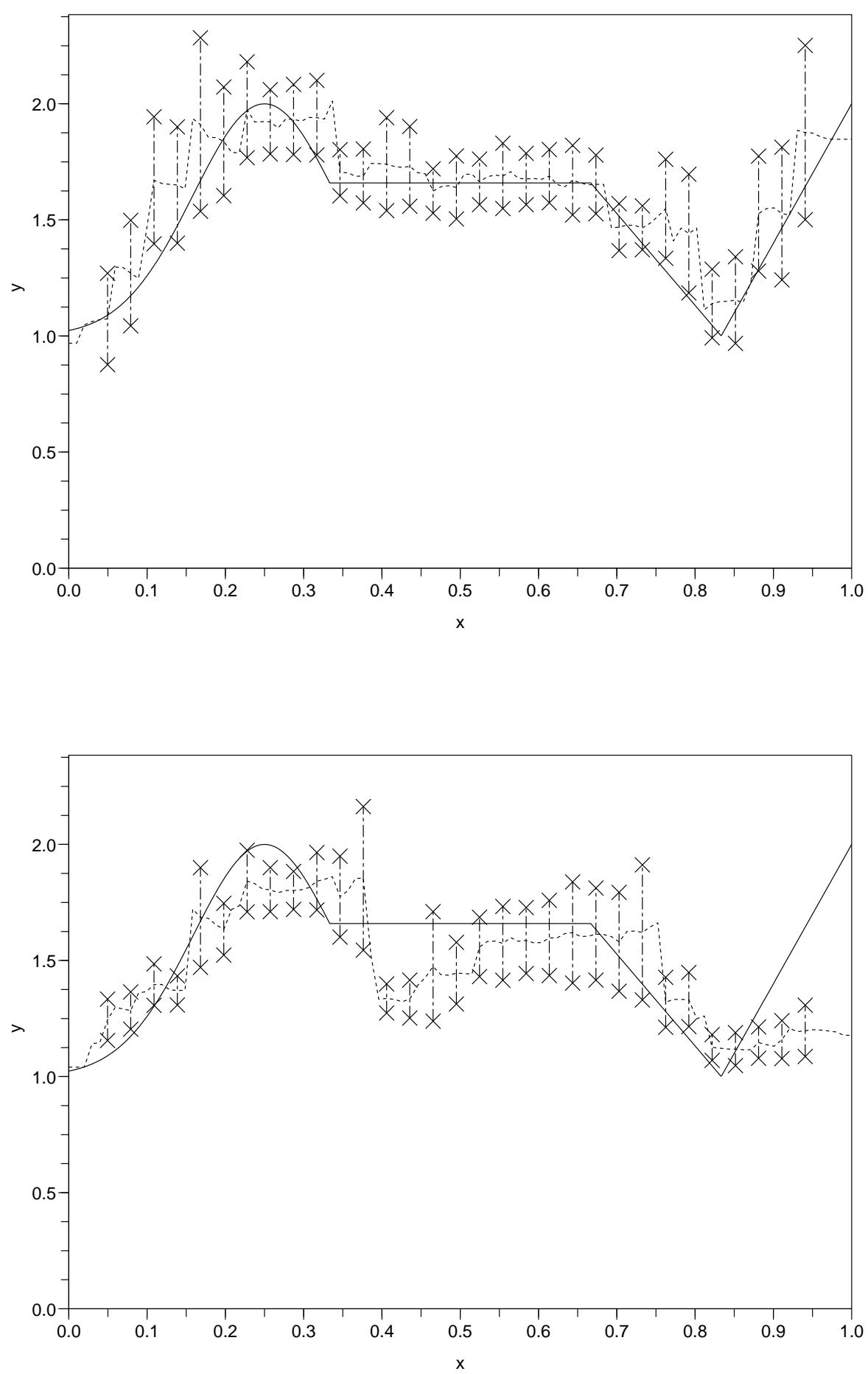

Figure 4: Case $d=1$ and $\alpha(x)=\alpha_{2}(x)$ : the frontier $g_{1}$ (solid line), its moment estimate $\widehat{g}_{n}$ (dotted line) with $a=3$, and $95 \%$ pointwise confidence intervals for $g_{1}$ obtained via Corollary 1 (dashed-dotted lines). Top: best situation, bottom: worst situation. 

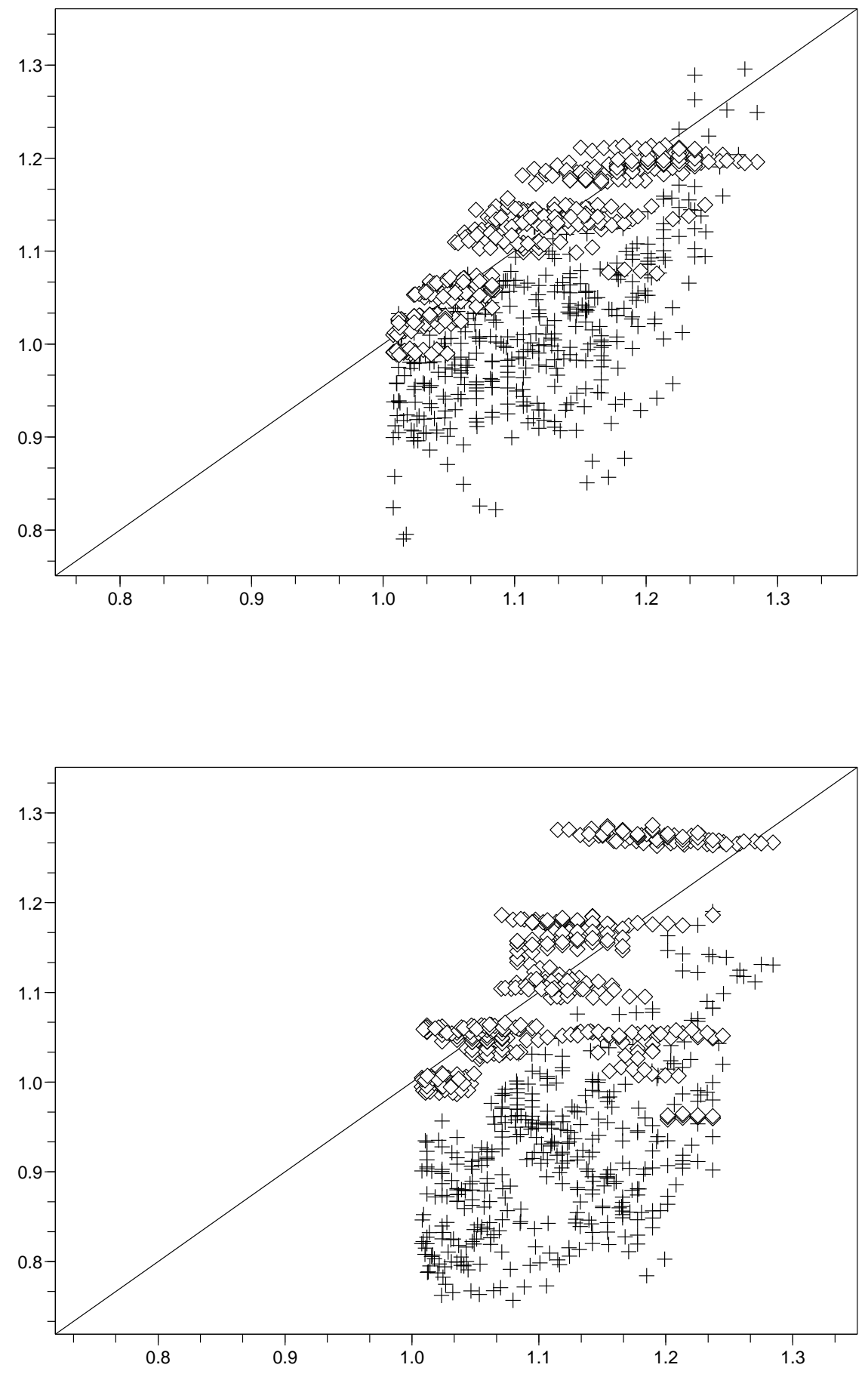

Figure 5: Case $d=2$ : pairs $\left(g\left(X_{i}\right), \widehat{g}\left(X_{i}\right)\right), i=1, \ldots, n$ associated to Girard-Jacob estimator $(+)$ and to the moment estimator $(\diamond)$ with $a=15$. The solid line has equation $y=x$. Top: best situation, bottom: worst situation. 